\title{
The Status of Exotic-quantum-number Mesons
}

\author{
C. A. Meyer ${ }^{1}$ and Y. Van Haarlem ${ }^{1}$ \\ ${ }^{1}$ Carnegie Mellon University, Pittsburgh, PA 15213
}

(Dated: October 31, 2018)

\begin{abstract}
The search for mesons with non-quark-antiquark (exotic) quantum numbers has gone on for nearly thirty years. There currently is experimental evidence of three isospin one states, the $\pi_{1}(1400)$, the $\pi_{1}(1600)$ and the $\pi_{1}(2015)$. For all of these states, there are questions about their identification, and even if some of them exist. In this article, we will review both the theoretical work and the experimental evidence associated with these exotic quantum number states. We find that the $\pi_{1}(1600)$ could be the lightest exotic quantum number hybrid meson, but observations of other members of the nonet would be useful.
\end{abstract}

PACS numbers: 14.40.-n,14-40.Rt,13.25.-k

\section{INTRODUCTION}

The quark model describes mesons as bound states of quarks and antiquarks $(q \bar{q})$, much akin to positronium $\left(e^{+} e^{-}\right)$. As described in Section II A, mesons have welldefined quantum numbers: total spin $J$, parity $P$, and $\mathrm{C}$ parity $C$, represented as $J^{P C}$. The allowed $J^{P C}$ quantum numbers for orbital angular momentum, $L$, smaller than three are given in Table II. Interestingly, for $J$ smaller than 3, all allowed $J^{P C}$ except $2^{--}$[1] have been observed by experiments. From the allowed quantum numbers in Table I] there are several missing combinations: $0^{--}, 0^{+-}, 1^{-+}$and $2^{+-}$. These are not possible for simple $q \bar{q}$ systems and are known as "exotic" quantum numbers. Observation of states with exotic quantum numbers has been of great experimental interest as it would be clear evidence for mesons beyond the simple $q \bar{q}$ picture.

\begin{tabular}{ccc|ccc|ccc}
\hline \hline$L$ & $S$ & $J^{P C}$ & $L$ & $S$ & $J^{P C}$ & $L$ & $S$ & $J^{P C}$ \\
\hline 0 & 0 & $0^{-+}$ & 1 & 0 & $1^{+-}$ & 2 & 0 & $2^{-+}$ \\
0 & 1 & $1^{--}$ & 1 & 1 & $0^{++}$ & 2 & 1 & $1^{--}$ \\
& & & 1 & 1 & $1^{++}$ & 2 & 1 & $2^{--}$ \\
& & 1 & 1 & $2^{++}$ & 2 & 1 & $3^{--}$ \\
\hline \hline
\end{tabular}

TABLE I. The allowed $J^{P C}$ quantum numbers for $q \bar{q}$ systems.

Moving beyond the simple quark-model picture of mesons, there have been predictions for states with these exotic quantum numbers. The most well known are $q \bar{q}$ states in which the gluons binding the system can contribute directly to the quantum numbers of the meson. However, other candidates include multi-quark states $(q \bar{q} q \bar{q})$ and states containing only gluons (glueballs). Early bag-model calculations [2] referred to states with $q \bar{q}$ and gluons as "hermaphrodite mesons", and predicted that the lightest nonet $\left(J^{P C}=1^{-+}\right)$might have masses near $1 \mathrm{GeV}$ as well as distinctive decay modes. They might also be relatively stable, and thus observable. While the name hermaphorodite did not survive, what are now known as "hybrid mesons" have become a very interesting theoretical and experimental topic and the status of these states, with particular emphasis on the exotic-quantum number ones is the topic of this article.
More information on meson spectroscopy in general can be found in a recent review by Klempt and Zaitsev [3]. Similarly, a recent review on the related topic of glueballs can be found in reference [4.

\section{THEORETICAL EXPECTATIONS FOR HYBRID MESONS}

\section{A. Mesons in The Quark Model}

In the quark model, mesons are bound states of quarks and antiquarks $(q \bar{q})$. The quantum numbers of such fermion-antifermion systems are functions of the total spin, $S$, of the quark-antiquark system, and the relative orbital angular momentum, $L$, between them. The spin $S$ and angular momentum $L$ combine to yield the total spin

$$
J=L \oplus S
$$

where $L$ and $S$ add as two angular momentums.

Parity is the result of a mirror reflection of the wave function, taking $\vec{r}$ into $-\vec{r}$. It can be written as

$$
P[\psi(\vec{r})]=\psi(-\vec{r})=\eta_{P} \psi(\vec{r}),
$$

where $\eta_{P}$ is the eigenvalue of parity. As application of parity twice must return the original state, $\eta_{P}= \pm 1$. In spherical coordinates, the parity operation reduces to the reflection of a $Y_{l m}$ function,

$$
Y_{l m}(\pi-\theta, \pi+\phi)=(-1)^{l} Y_{l m}(\theta, \phi) .
$$

From this, we conclude that $\eta_{P}=(-1)^{l}$.

For a $q \bar{q}$ system, the intrinsic parity of the antiquark is opposite to that of the quark, which yields the total parity of a $q \bar{q}$ system as

$$
P(q \bar{q})=-(-1)^{L} .
$$

Charge conjugation, $C$, is the result of a transformation that takes a particle into its antiparticle. For a $q \bar{q}$ system, only electrically-neutral states can be eigenstates of $C$. In order to determine the eigenvalues of $C\left(\eta_{C}\right)$, 
we need to consider a wave function that includes both spatial and spin information

$$
\Psi(\vec{r}, \vec{s})=R(r) Y_{l m}(\theta, \phi) \chi(\vec{s}) .
$$

As an example, we consider a $u \bar{u}$ system, the $C$ operator acting on this reverses the meaning of $u$ and $\bar{u}$. This has the effect of mapping the vector $\vec{r}$ to the $u$ quark into $-\vec{r}$. Thus, following the arguments for parity, the spatial part of $C$ yields a factor of $(-1)^{L}$. The spin wave function also reverse the two individual spins. For a symmetric $\chi$, we get a factor of 1 , while for an antisymmetric $\chi$, we get a factor of -1 . For two spin $\frac{1}{2}$ particles, the $S=0$ singlet is antisymmetric, while the $S=1$ triplet is symmetric. Combining all of this, we find that the C-parity of (a neutral) $q \bar{q}$ system is

$$
C(q \bar{q})=(-1)^{L+S} .
$$

Because $C$-parity is only defined for neutral states, it is useful to extend this to the more general $G$-parity which can be used to describe all $q \bar{q}$ states, independent of charge. For isovector states $(I=1), C$ would transform a charged member into the oppositely charged state (e.g. $\pi^{+} \rightarrow \pi^{-}$). In order to transform this back to the original charge, we would need to perform a rotation in isospin $\left(\pi^{-} \rightarrow \pi^{+}\right)$. For a state of whose neutral member has $C$-parity $C$, and whose total isospin is $I$, the $G$-parity is defined to be

$$
G=C \cdot(-1)^{I},
$$

which can be generalized to

$$
G(q \bar{q})=(-1)^{L+S+I} .
$$

The latter is valid for all of the $I=0$ and $I=1$ members of a nonet. This leads to mesons having well defined quantum numbers: total angular momentum, $J$, isospin, $I$, parity $P$, C-parity, $C$, and G-parity, $G$. These are represented as $\left(I^{G}\right) J^{P C}$, or simply $J^{P C}$ for short. For the case of $L=0$ and $S=0$, we have $J^{P C}=0^{-+}$, while for $L=0$ and $S=1, J^{P C}=1^{--}$. The allowed quantum numbers for $L$ smaller than three are given in Table 1 .

\section{B. Notation and Quantum Numbers of Hybrids}

The notation for hybrid mesons we use is that from the Particle Data Group (PDG) 1]. In the PDG notation, the parity and charge conjugation determine the name of the hybrid, which is taken as the name of the normal meson of the same $J^{P C}$ and isospin. The total spin is then used as a subscript to the name. While various models predict different nonets of hybrid mesons, the largest number of nonets is from the flux-tube model (see Section IIC). For completeness, we list all of these as well as their PDG names in Table II. The first entry is the isospin one $(I=1)$ state. The second and third are those with isospin equal to zero $(I=0)$ and the fourth is the kaon-like state with isospin one-half $\left(I=\frac{1}{2}\right)$. In the case of the $I=0$ states, the first is taken as the mostly $u \bar{u}$ and $d \bar{d}$ state (so-called $n \bar{n}$ ), while the second is mostly $s \bar{s}$. For the $I=0$ states, $C$-parity is well defined, but for $I=1$, only the neutral member can have a defined $C$ parity. However, the more general $G$-parity can be used to describe all of the $I=1$ members (see equation 8). Thus, the $G$-parity can be used to identify exotic quantum numbers, even for charged $I=1$ members of a nonet. For the case of the kaon-like states, neither $C$-parity nor $G$-parity is defined. Thus, the $I=\frac{1}{2}$ members of a nonet can not have explicitly-exotic quantum numbers.

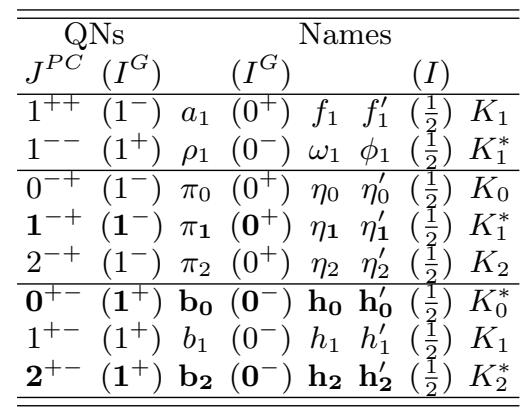

TABLE II. The naming scheme for hybrid mesons. The first state listed for a given quantum number is the isospin one state. The second state is the isospin zero state that is mostly $u$ and $d$ quarks $(n \bar{n})$, while the third name is for the mostly $s \bar{s}$ isospin zero state. Note that for the kaons, the $C$ - and $G$-parity are not defined. Kaons cannot not have manifestly exotic quantum numbers. States that have exotic quantum numbers are shown in bold.

In Table III we show the $J^{P}$ of the three exotic $I=$ 1 mesons from Table II. We also show the normal $(q \bar{q})$ meson of the same $J^{P}$ and the $I^{G}$ quantum numbers for these states. The exotic mesons have the opposite $G$-parity relative to the normal meson. This provides a simple mechanism for identifying if a charged $I=1$ state has exotic quantum numbers.

\begin{tabular}{ccccc}
\hline \hline$J^{P}$ & normal meson exotic meson \\
& name & $\left(I^{G}\right)$ & name & $\left(I^{G}\right)$ \\
\hline $0^{+}$ & $a_{0}$ & $\left(1^{-}\right)$ & $b_{0}$ & $\left(1^{+}\right)$ \\
$1^{-}$ & $\rho$ & $\left(1^{+}\right)$ & $\pi_{1}$ & $\left(1^{-}\right)$ \\
$2^{+}$ & $a_{2}$ & $\left(1^{-}\right)$ & $b_{2}$ & $\left(1^{+}\right)$ \\
\hline \hline
\end{tabular}

TABLE III. The $J^{P}$ and $I^{G}$ quantum numbers for the exotic mesons and the normal mesons of the same $J^{P}$.

\section{Model Predictions}

The first predictions for exotic quantum number mesons came from calculations in the Bag model [5, 6]. In this model, boundary conditions are placed on quarks and gluons confined inside a bag. A hybrid meson is formed by combining a $q \bar{q}$ system (with spin 0 or 1 ) with a transverse-electric (TE) gluon $\left(J^{P C}=1^{+-}\right)$. This 
yields four nonets of hybrid mesons with quantum numbers $J^{P C}=1^{--}, 0^{-+}, 1^{-+}$and $2^{-+}$. These four nonets are roughly degenerate in mass and early calculations predicted the mass of a $1^{-+}$to be in the range of 1.2 to $1.4 \mathrm{GeV}$ 7, 8. In the bag model, the transverse-magnetic gluon is of higher mass. It has $J^{P C}=1^{-+}$and combined with the same $S=0$ and $S=1 q \bar{q}$ systems yield four additional nonets with $J^{P C}=1^{++}, 0^{+-}, 1^{+-}$and $2^{+-}$. These would presumably be heavier than the nonets built with the TE gluon.

Another method that has been used to predict the hybrid masses are "QCD spectral sum rules" (QSSR). Using QSSR, one examines a two-point correlator of appropriate field operators from QCD and produces a sum rule by equating a dispersion relation for the correlator to an operator product expansion. QSSR calculations initially found a $1^{-+}$state near $1 \mathrm{GeV}$ 9, 10]. A $0^{--}$state was also predicted around $3.8 \mathrm{GeV}$ in mass [10. Newer calculations 11] tend to favor a $1^{-+}$hybrid mass in the range of 1.6 to $2.1 \mathrm{GeV}$, and favor the $\pi_{1}(1600)$ (see Section III C) as the lightest exotic hybrid. Recently, Narison 12] looked at the calculations for $J^{P C}=1^{-+}$states with particular emphasis in understanding differences in the results between QSSR and Lattice QCD calculations (see Section IID). He found that the $\pi_{1}(1400)$ and $\pi_{1}(1600)$ may be consistent with 4-quark states, while QSSR are consistent with the $\pi_{1}(2015)$ (see Section III D) being the lightest hybrid meson.

The formation of flux tubes was first introduced in the 1970's by Yoichiro Nambu 13, 14 to explain the observed linear Regge trajectories - the linear dependence of mass squared, $\mathrm{m}^{2}$, of hadrons on their spin, $J$. This linear dependence results if one assumes that mass-less quarks are tied to the ends of a relativistic string with constant mass (energy) per length and the system rotating about its center. The linear $m^{2}$ versus $J$ dependence only arises when the mass density per length is constant, which is equivalent to a linear potential.

In the heavy-quark sector, lattice QCD [15] calculations show a distribution of the gluonic field (action density) which is mostly confined to the region between the quark and the antiquark. A picture which is very similar to that inspired by the "flux-tube model". Within the flux-tube model [16, 17, one can view hybrids as mesons with angular momentum in the flux tube. Naively, one can imagine two degenerate excitations, one with the tube going clockwise and one counter clockwise. It is possible to write linear combinations of these that have definite spin, parity and C-parity. For the case of one unit of angular momentum in the tube, the flux tube behaves as if it has quantum numbers $J^{P C}=1^{+-}$or $1^{-+}$. The basic quantum numbers of hybrids are obtained by adding the tube's quantum numbers to that of the underlying meson.

In the flux-tube model, the tube carries angular momentum, $m$, which then leads to specific predictions for the product of $C$-parity and parity $(C P)$. For $m=0$, one has $C P=(-1)^{S+1}$, while for the first excited states, $(m=1)$, we find that $C P=(-1)^{S}$. The excitations are then built on top of the $s$-wave mesons, $(L=0)$, where the total spin can be either $S=0$ or $S=1$. For the case of $m=0$, we find $C P$ as follows,

$$
\left.(m=0) \begin{array}{cc}
S=0 & 0^{-+} \\
S=1 & 1^{--}
\end{array}\right\} \begin{gathered}
(-1)^{L+1}(-1)^{S+L}=(-1)^{S+1} \\
\text { Normal Mesons }
\end{gathered}
$$

which are the quantum numbers of the normal, $q \bar{q}$, mesons as discussed in Section IIA. For the case of $m=1$, where we have one unit of angular momentum in the flux tube, we find the following $J^{P C}$ quantum numbers

$$
\left.(m=1) \begin{array}{cc}
S=0 & 0^{-+} \\
S=1 & 1^{--}
\end{array}\right\} 0^{-+}, \mathbf{0}^{+-}, \mathbf{1}^{-+}, 1^{+-}, 2^{-+}, \mathbf{2}^{+-} .
$$

The resulting quantum numbers are obtained by adding both $1^{+-}$and $1^{-+}$to the underlying $q \bar{q}$ quantum numbers $\left(0^{-+}\right.$and $\left.1^{--}\right)$.

From the two $L=0$ meson nonets, we expect eight hybrid nonets, (72 new mesons!). Two of these nonets arise from the $q \bar{q}$ in an $S=0$ (singlet) state, while six arise for the $q \bar{q}$ in the $S=1$ (triplet) state. Of the six states built on the triplet $q \bar{q}$, three have exotic quantum numbers (as indicated in bold above).

In the picture presented by the flux-tube model, the hybrids are no different than other excitations of the $q \bar{q}$ states. In addition to "orbital" and "radial" excitations, we also need to consider "gluonic" excitations. Thus, the flux-tube model predicts eight nonets of hybrid mesons $\left(0^{+-}, 0^{-+}, 1^{++}, 1^{--}, 1^{-+}, 1^{+-}, 2^{-+}\right.$and $\left.2^{+-}\right)$. The model also predicts that all eight nonets are degenerate in mass, with masses expected near $1.9 \mathrm{GeV}$ [17].

An alternate approach to calculating properties of hybrid mesons comes from the effective QCD Coulombgauge Hamiltonian. Here, Foch states for hadrons are constructed from the vacuum as well as quark and gluon operators. In this model, the lightest hybrid nonets are $J^{P C}=1^{+-}, 0^{++}, 1^{++}$and $2^{++}$, none of which are exotic. The first excitation of these $(L=1)$, yields the nonets $1^{-+}, 3^{-+}$and $0^{--}$, all of which are exotic [18, 19]. In this model, the $1^{-+}$is the lightest exotic quantum number hybrid, with a mass in the range of 2.1 to $2.3 \mathrm{GeV}$. Predictions are also made for the lightest $c \bar{c}$ exotic hybrid, which is found in the range of 4.1 to $4.3 \mathrm{GeV}$.

In Table IV] are presented a summary of the mass predictions for the various model calculations for hybrid meson masses.

\begin{tabular}{clc}
\hline Mass $(\mathrm{GeV})$ & Model & \multicolumn{2}{c}{ Reference } \\
\hline $1.0-1.4$ & Bag Model & {$[2,5,6]$} \\
$1.0-1.9$ & QSSR & $[9]-12]$ \\
$1.8-2.0$ & Flux Tube & {$[17$} \\
$2.1-2.3$ & Hamiltonian & {$[18$} \\
\hline \hline
\end{tabular}

TABLE IV. Mass predictions for hybrid mesons from various models. 


\section{Lattice Predictions}

Lattice QCD (LQCD) calculations may provide the most accurate estimate to the masses of hybrid mesons. While these calculations have progressively gotten better, they are still limited by a number of systematic effects. Currently, the most significant of these is related to the mass of the light quarks used in the calculations. This is typically parametrized as the pion mass, and extrapolations need to be made to reach the physical pion mass. This is often made as a linear approximation, which may not be accurate. In addition, as the the quark mass becomes lighter, two-meson decay channels become possible. These may distort the resulting spectrum.

Most calculations have been performed with what is effectively the strange-quark mass. However, it may not be safe to assume that this is the mass of the $s \bar{s}$ member of the nonet, and one needs to be aware of the approximations made to move the estimate to the $u \bar{u} / d \bar{d}$ mass. The bottom line is that no one would be surprised if the true hybrid masses differed by several hundred $\mathrm{MeV}$ from the best predictions.

\begin{tabular}{ccc}
\hline \hline Author & \multicolumn{2}{c}{$1^{-+}$Mass $\left(\mathrm{GeV} / \mathrm{c}^{2}\right)$} \\
Collab. & $u \bar{u} / d \bar{d}$ & $s \bar{s}$ \\
\hline UKQCD [20] & $1.87 \pm 0.20$ & $2.0 \pm 0.2$ \\
MILC [21] & $1.97 \pm 0.09 \pm 0.30$ & $2.170 \pm 0.080 \pm 0.30$ \\
SESAM [22] & $1.9 \pm 0.20$ & \\
MILC [23] & $2.11 \pm 0.10 \pm($ sys $)$ & \\
Mei 24] & $2.013 \pm 0.026 \pm 0.071$ & \\
Hedditch [25] & $1.74 \pm 0.25$ & \\
Bernard [26] & $1.792 \pm 0.139$ & $2.100 \pm 0.120$ \\
McNeile [27] & $2.09 \pm 0.1$ & \\
\hline \hline
\end{tabular}

TABLE V. Recent results for the light-quark $1^{-+}$hybrid meson masses.

While the flux-tube model (see Section II C predicts that the lightest eight nonets of hybrid mesons are degenerate in mass at about $1.9 \mathrm{GeV}$, LQCD calculations consistently show that the $J^{P C}=1^{-+}$nonet is the lightest. Predictions for the mass of this state have varied from 1.8 to $2.1 \mathrm{GeV}$, with an average about in the middle of these. Table $\mathrm{V}$ shows a number of these predictions made over the last several years. Most of these 20]25] were made in the quenched approximation (no $q \bar{q}$ loops allowed in the quenched calculation), while newer calculations [26 29] are dynamic (not quenched).

However, the masses in Table $\mathrm{V}$ may not be the best approximations to the hybrid masses. It has been noted [30] that Table V] is not a very useful way of displaying the results. Rather, the mass needs to be correlated with the light-quark mass used in the calculation. This is usually represented as the pion mass. In Figure 1 are shown the predictions from the same groups as a function of the pion masses used in their calculations. In order to obtain the hybrid mass, one needs to extrapolate to the physical pion mass.

There are fewer predictions for the masses of the other

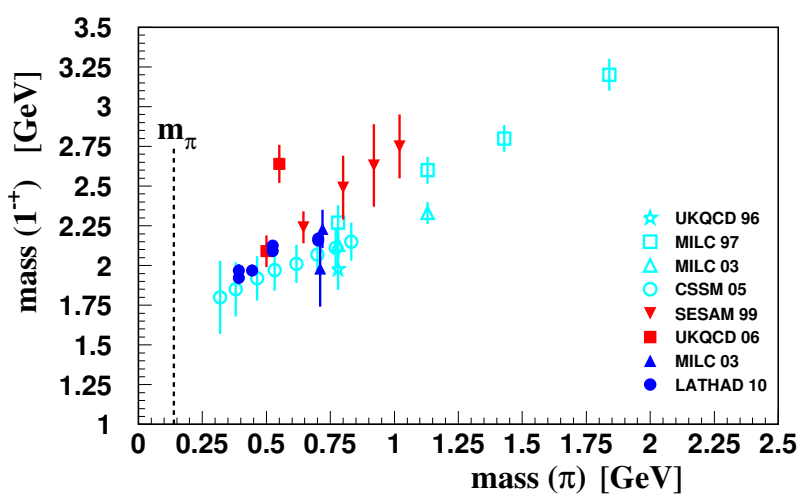

FIG. 1. (Color on line.) The mass of the $J^{P C}=1^{-+}$exotic hybrid as a function of the pion mass from lattice calculations. The open (cyan) symbols correspond to quenched calculations, while the solid (red and blue) symbols are dynamic (unquenched) calculations: open (cyan) star [20], open (cyan) squares 21, open (cyan) upright triangles [26], open (cyan) circles 25], solid (red) downward triangles [22], solid (red) squares [27], solid (blue) upright triangles [26] and solid (blue) circles [29].

exotic-quantum number states. Bernard 21 calculated the splitting between the $0^{+-}$and the $1^{-+}$state to be about $0.2 \mathrm{GeV}$ with large errors. A later calculation using a clover action 23 found a splitting of $0.270 \pm 0.2 \mathrm{GeV}$. The SESAM collaboration 22] has one such calculation, the results of which are shown in Table VI.

\begin{tabular}{ccc}
\hline \hline Multiplet & $J^{P C}$ & Mass \\
\hline$\pi_{1}$ & $1^{-+}$ & $1.9 \pm 0.2 \mathrm{GeV} / \mathrm{c}^{2}$ \\
$b_{2}$ & $2^{+-}$ & $2.0 \pm 1.1 \mathrm{GeV} / c^{2}$ \\
$b_{0}$ & $0^{+-}$ & $2.3 \pm 0.6 \mathrm{GeV} / c^{2}$ \\
\hline \hline
\end{tabular}

TABLE VI. Estimates of the masses of exotic quantum number hybrids 22].

A significant LQCD calculation has recently been performed which predicts the entire spectrum of light-quark isovector mesons [28, 29]. The fully dynamical (unquenched) calculation is carried out with two flavors of the lightest quarks and a heavier third quark tuned to the strange quark mass. Calculations are performed on two lattice volumes and using four different masses for the lightest quarks - corresponding to pion masses of 700 , 520, 440 and $390 \mathrm{MeV}$. In the heaviest case, the lightest quark masses are the same at the strange mass. The computed spectrum of isovector states for this heavy case is shown in Figure 2 (where the mass is plotted as a ratio to the $\Omega$-baryon mass $(1.672 \mathrm{GeV}))$. In the plot, the right-most columns correspond to the exotic $\pi_{1}, b_{0}$ and $b_{2}$ states. Interestingly, the $1^{-+} \pi_{1}$ is the lightest, and both a ground state and what appears to be an excited state are predicted. The other two exotic-quantum-number states appear to be somewhat heavier than the $\pi_{1}$ with an excited state for the $b_{2}$ visible.

In addition to performing the calculation near the 


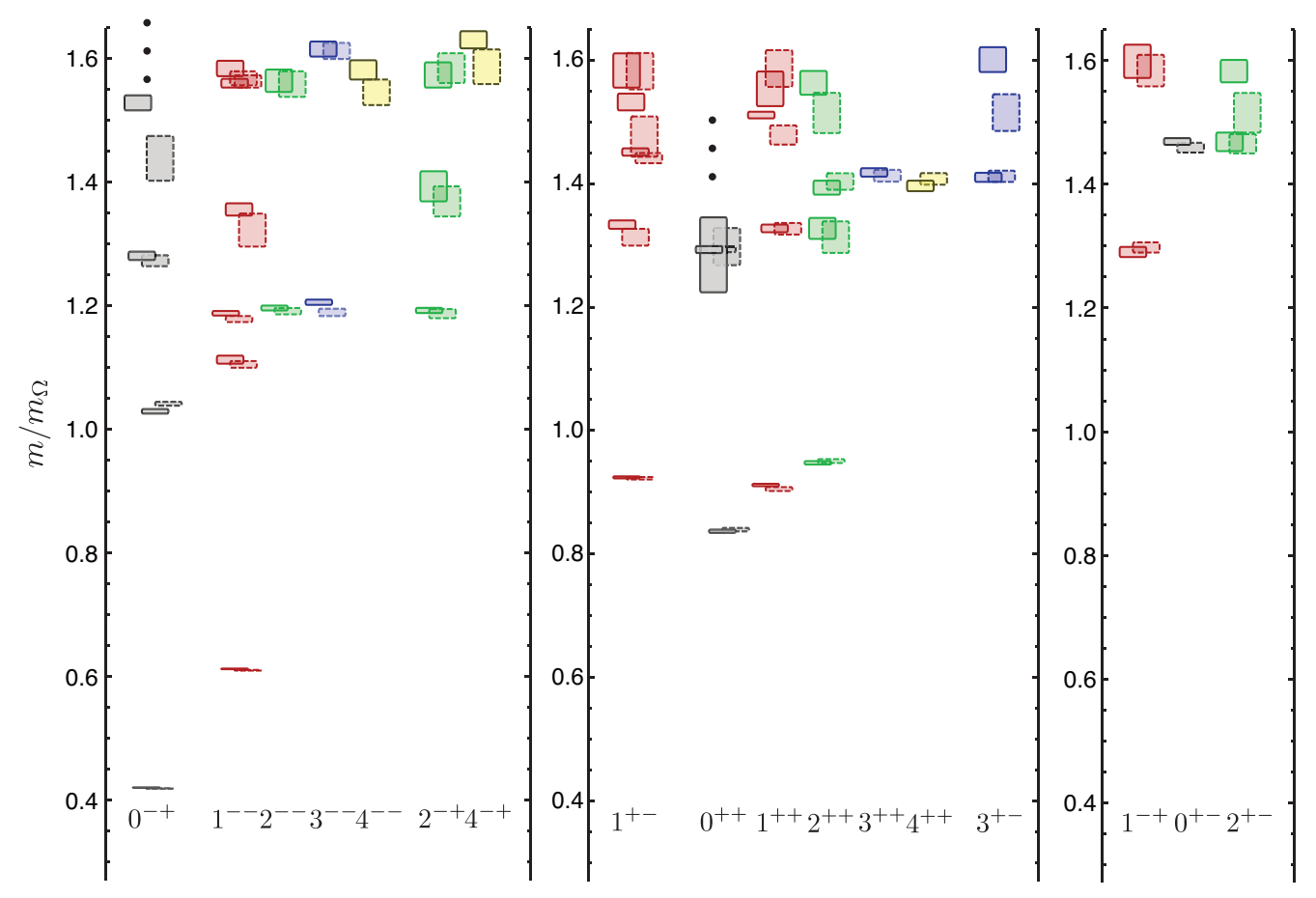

FIG. 2. (Color on line) The LQCD prediction for the spectrum of isovector mesons. The quantum numbers are listed across the bottom, while the color denotes the spin. Solid (dashed) bordered boxes on a $2.0^{3}\left(2.4^{3}\right)$ fm volume lattice, little volume dependence is observed. The three columns at the far right are exotic-quantum numbers. The plot is taken from reference 29$]$

physical quark mass, there are a number of important innovations. First, the authors have found that the reduced rotational symmetry of a cubic lattice can be overcome on sufficiently fine lattices. They used meson operators of definite continuum spin subduced into the irreducible representations of cubic rotations and observed very strong correlation between operators and the spin of the state. In this way they were able to make spin assignments from a single lattice spacing. Second, the unprecedented size of the operator basis used in a variational calculation allowed the extraction of many excited states with confidence.

There were also phenomenological implications of these lattice results. A subset of the meson operators feature the commutator of two gauge-covariant derivatives, equal to the field-strength tensor, which is non-zero only for non-trivial gluonic field configurations. Large overlap onto such operators was used to determine the degree to which gluonic excitations are important in the state, i.e., what one would call the hybrid nature of the state. In particular, the exotic quantum number states all have large overlap with this type of operator, a likely indication of hybrid nature over, say, multiquark structure. In addition to the exotic-quantum number states, several normal-quantum-number states also had large overlap with the non-trivial gluonic field. In particular, states with $J^{P C}=1^{--}, 2^{-+}$with approximately the same mass as the lighter $1^{-+}$state were noted.

In order to extract the masses of states, it is necessary to work at the physical pion mass. While work is currently underway to extract a point at $m_{\pi} \approx 280 \mathrm{MeV}$, this limit has not yet been reached. To attempt to extrapolate, one can plot the extracted state masses as a function of the pion mass squared, which acts as a proxy for the light quark mass (see Figure 3). While linearly extrapolating to the physical pion mass ignores constraints from chiral dynamics, it is probably safe to say that both the $\pi_{1}(1600)$ and the $\pi_{1}(2015)$ (as discussed below) could be consistent with the expected $1^{-+}$mass. They are also consistent with the ground and first-excited $\pi_{1}$ state. It appears that the $b_{0}$ and $b_{2}$ masses will likely be several hundred $\mathrm{MeV}$ heavier than the lightest $\pi_{1}$.

Lattice calculations have also been performed to look for other exotic quantum number states. Bernard 21] included operators for a $0^{--}$state, but found no evidence for a state with these quantum numbers in their quenched calculation. Dudek et a. 29] looked for both $0^{--}$and $3^{-+}$states in their lattice data. They found some evidence for states with these quantum numbers, but the lightest masses were more than $2 \mathrm{GeV}$ above the mass of the $\rho$ meson.

These recent lattice calculations are extremely promising. They reaffirm that hybrid mesons form part of the low-energy QCD spectrum and that exotic quantum number states exist. They also provide, for the first time, the possibility of assessing the gluonic content of a calculated lattice state. Similar calculations are currently underway for the isoscalar sector where preliminary re- 


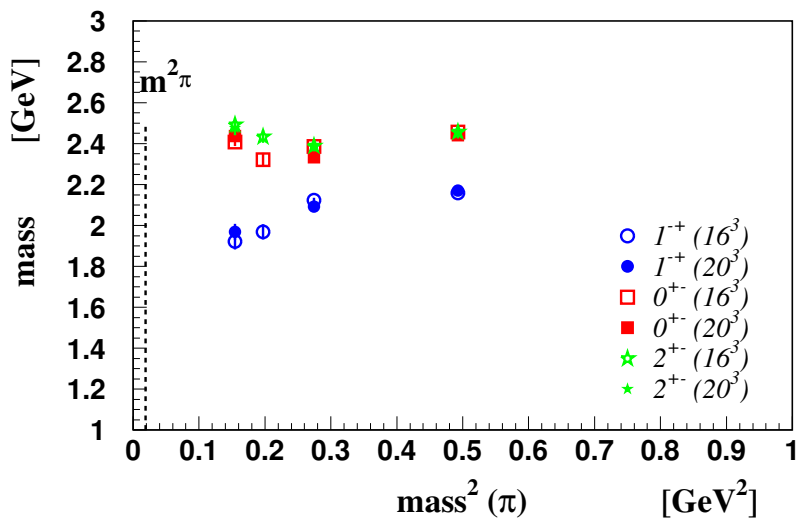

FIG. 3. (Color on line) The mass spectrum of the three exotic quantum number states 29 . The open figures are for a $16^{3}$ spatial dimension lattice, while the solid are for a $20^{3}$ spatial lattice. The (blue) circles are the mass of the $1^{-+}$state, the (green) squares are the mass of the $0^{+-}$state and the (red) stars are the $2^{+-}$state.

sults 30 for the mass scale appear consistent with those shown here in the isovector sector. These calculations will also extract the flavor mixing angle, an important quantity for phenomenology.

\section{E. Decay Modes}

Currently, decays of hybrid mesons can only be calculated within models. Such models exist, having been developed to compute the decays of normal mesons. A basic feature of these is the so-called triplet-P-zero $\left({ }^{3} P_{0}\right)$ model. In the ${ }^{3} P_{0}$ model, a meson decays by producing a $q \bar{q}$ pair with vacuum quantum numbers $\left(J^{P C}=0^{++}\right)$.

A detailed study by Ackleh, Barnes and Swanson 31. established that the ${ }^{3} P_{0}$ amplitudes are dominant in most light-quark meson decays. They also determined the parameters in decay models by looking at the well known decays of mesons. This work was later extended to provide predictions for the decay of all orbital and radial excitations of mesons lighter than $2.1 \mathrm{GeV}$ [32]. This tour-de-force in calculation has served as the backdrop against which most light-quark meson and hybrid candidates are compared.

The original calculations for the decays of hybrids in the flux-tube model were carried out by Isgur [17. Within their model, Close and Page [33, confirmed the results and expanded the calculations to include additional hybrids. Using improved information about mesons and using simple harmonic oscillator $(\mathrm{SHO})$ wave functions, they were able to compute the decay width of hybrid mesons. They also provided arguments for the selection rule that hybrids prefer to decay to an $L=0$ and an $L=1$ meson. The suppression of a pair of $L=0$ mesons arises in the limit that the two mesons have the same inverse radius in the Simple Harmonic Oscillator wave functions. Thus, these decays are not strictly for-

\begin{tabular}{ccccc}
\hline \hline \multicolumn{2}{c}{ Name } & \multicolumn{2}{c}{$\mathbf{J}^{\mathbf{P C}}$ Total Width $\mathrm{MeV}$} & Large Decays \\
& & PSS & IKP & \\
\hline$\pi_{1}$ & $1^{-+}$ & $81-168$ & 117 & $b_{1} \pi, \rho \pi, f_{1} \pi, a_{1} \eta$, \\
& & & & $\eta(1295) \pi, K_{1}^{A} K, K_{1}^{B} K$ \\
$\eta_{1}$ & $1^{-+}$ & $59-158$ & 107 & $a_{1} \pi, f_{1} \eta, \pi(1300) \pi$, \\
& & & & $K_{1}^{A} K, K_{1}^{B} K$ \\
$\eta_{1}^{\prime}$ & $1^{-+}$ & $95-216$ & 172 & $K_{1}^{B} K, K_{1}^{A} K, K^{*} K$ \\
\hline$b_{0}$ & $0^{+-}$ & $247-429$ & 665 & $\pi(1300) \pi, h_{1} \pi$ \\
$h_{0}$ & $0^{+-}$ & $59-262$ & 94 & $b_{1} \pi, h_{1} \eta, K(1460) K$ \\
$h_{0}^{\prime}$ & $0^{+-}$ & $259-490$ & 426 & $K(1460) K, K_{1}^{A} K, h_{1} \eta$ \\
\hline$b_{2}$ & $2^{+-}$ & $5-11$ & 248 & $a_{2} \pi, a_{1} \pi, h_{1} \pi$ \\
$h_{2}$ & $2^{+-}$ & $4-12$ & 166 & $b_{1} \pi, \rho \pi$ \\
$h_{2}^{\prime}$ & $2^{+-}$ & $5-18$ & 79 & $K_{1}^{B} K, K_{1}^{A} K, K_{2}^{*} K, h_{1} \eta$ \\
\hline \hline
\end{tabular}

TABLE VII. Exotic quantum number hybrid width and decay predictions from reference [34]. The column labeled PSS (Page, Swanson and Szczepaniak) is from their model, while the IKP (Isgur, Karl and Paton) is their calculation of the model in reference [17. The variations in width for PSS come from different choices for the masses of the hybrids. The $K_{1}^{A}$ represents the $K_{1}(1270)$ while the $K_{1}^{B}$ represents the $K_{1}(1400)$.

bidden, but are suppressed depending on how close the two inverse radii are. This led to the often-quoted predication for the decays of the $\pi_{1}$ hybrid given in equation 9 .

$$
\begin{gathered}
\pi b_{1}: \pi f_{1}: \pi \rho: \eta \pi: \pi \eta^{\prime} \\
= \\
170: 60: 5-20: 0-10: 0-10
\end{gathered}
$$

The current predictions for the widths of exoticquantum-number hybrids are based on model calculations by Page et al. 34] for which the results are given in Table VII. They also computed decay rates for the hybrids with normal $q \bar{q}$ quantum numbers (results in Table VIII). While a number of these states are expected to be broad (in particular, most of the $0^{+-}$exotic nonet), states in both the $2^{+-}$and the $1^{-+}$nonets are expected to have much narrower widths. The expected decay modes involve daughters that in turn decay. Thus making the overall reconstruction and analysis of these states much more complicated then simple two-pseudoscalar decays.

For the non-exotic quantum numbers states, it will be even more difficult. They are likely to mix with nearby normal $q \bar{q}$ states, complicating the expected decay pattern for both the hybrid and the normal mesons. However, the decays in Table VIII can be used as a guideline to help in identifying these states. In searches for hybrid mesons, the nonets with exotic quantum numbers provide the cleanest environment in which to search for these objects.

Close and Thomas 35] reexamined this problem in terms of work on hadronic loops in the $c \bar{c}$ sector by Barnes and Swanson 36. They conclude that in the limit where all mesons in a loop belong to a degenerate subset, vector hybrid mesons remain orthogonal to the 
$q \bar{q}$ states $\left(J^{P C}=11^{--}{ }^{3} S_{1}\right.$ and $\left.{ }^{3} D_{1}\right)$ and mixing may be minimal. Thus, the search for hybrids with vector $q \bar{q}$ quantum numbers may not be as difficult as the other non-exotic quantum number hybrids.

\begin{tabular}{|c|c|c|c|c|}
\hline \multirow[t]{2}{*}{ Particle } & \multirow[t]{2}{*}{$\mathbf{J}^{\mathrm{PC}}$} & \multicolumn{2}{|c|}{ Total Width $\mathrm{MeV}$} & \multirow[t]{2}{*}{ Large Decays } \\
\hline & & PSS & IKP & \\
\hline$\rho_{1}$ & $1^{--}$ & $70-121$ & 112 & $a_{1} \pi, \omega \pi, \rho \pi$ \\
\hline$\omega_{1}$ & $1^{--}$ & $61-134$ & 60 & $\rho \pi, \omega \eta, \rho(1450) \pi$ \\
\hline$\phi_{1}$ & $1^{--}$ & $95-155$ & 120 & $K_{1}^{B} K, K^{*} K, \phi \eta$ \\
\hline$a_{1}$ & $1^{++}$ & $108-204$ & 269 & $\rho(1450) \pi, \rho \pi, K^{*} K$ \\
\hline$h_{1}$ & $1^{++}$ & $43-130$ & 436 & $K^{*} K, a_{1} \pi$ \\
\hline$h_{1}^{\prime}$ & $1^{++}$ & $119-164$ & 219 & $K^{*}(1410) K, K^{*} K$ \\
\hline$\pi_{0}$ & $0^{-+}$ & $102-224$ & 132 & $\rho \pi, f_{0}(1370) \pi$ \\
\hline$\eta_{0}$ & $0^{-+}$ & $81-210$ & 196 & $a_{0}(1450) \pi, K^{*} K$ \\
\hline$\eta_{0}^{\prime}$ & $0^{-+}$ & $215-390$ & 335 & $K_{0}^{*} K, f_{0}(1370) \eta, K^{*} K$ \\
\hline$b_{1}$ & $1^{+-}$ & $177-338$ & 384 & $\omega(1420) \pi, K^{*} K$ \\
\hline$h_{1}$ & $1^{+-}$ & $305-529$ & 632 & $\rho(1450) \pi, \rho \pi, K^{*} K$ \\
\hline$h_{1}^{\prime}$ & $1^{+-}$ & $301-373$ & 443 & $K^{*}(1410) K, \phi \eta, K^{*} K$ \\
\hline$\pi_{2}$ & $2^{-+}$ & $27-63$ & 59 & $\rho \pi, f_{2} \pi$ \\
\hline$\eta_{2}$ & $2^{-+}$ & $27-58$ & 69 & $a_{2} \pi$ \\
\hline$\eta_{2}^{\prime}$ & $2^{-+}$ & $38-91$ & 69 & $K_{2}^{*} K, K^{*} K$ \\
\hline
\end{tabular}

TABLE VIII. Non-exotic quantum number hybrid width and decay predictions from reference 34. The column labeled PSS (Page, Swanson and Szczepaniak) is from their model, while the IKP (Isgur, Karl and Paton) is their calculation of the model in reference [17. The variations in width for PSS come from different choices for the masses of the hybrids. The $K_{1}^{A}$ represents the $K_{1}(1270)$ while the $K_{1}^{B}$ represents the $K_{1}(1400)$.

Almost all models of hybrid mesons predict that they will not decay to identical pairs of mesons. Many also predict that decays to pairs of $L=0$ mesons will be suppressed, leading to decays of an $(L=0)(L=1)$ pair as the favored hybrid decay mode. Page [37] undertook a study of these models of hybrid decay that included "TE hybrids" ( with a transverse electric constituent gluon) in the bag model as well as "adiabatic hybrids" in the flux-tube model (hybrids in the limit where quarks move slowly with respect to the gluonic degrees of freedom). In such cases, the decays to pairs of orbital angular momentum $L=0$ (Swave) mesons were found to vanish. In both cases, it had been noted that this was true when the quark and the antiquark in the hybrid's daughters have identical constituent masses with the same $S$-wave spatial wave functions, and the quarks are non-relativistic. In order to understand this, Page looked for an underlying symmetry that could be responsible for this. He found that symmetrization of connected decay diagrams (see Figure 4(a)) where the daughters are identical except for flavor and spin vanish when equation 10 is satisfied.

$$
C_{A}^{0} P_{A}=(-1)^{\left(S_{A}+S_{q \bar{q}}+1\right)}
$$

For meson $A$ decaying to daughters $B$ and $C, C_{A}^{0}$ is the C-parity of the neutral isospin member of the decaying meson $A, P_{A}$ is its parity and $S_{A}$ is its intrinsic

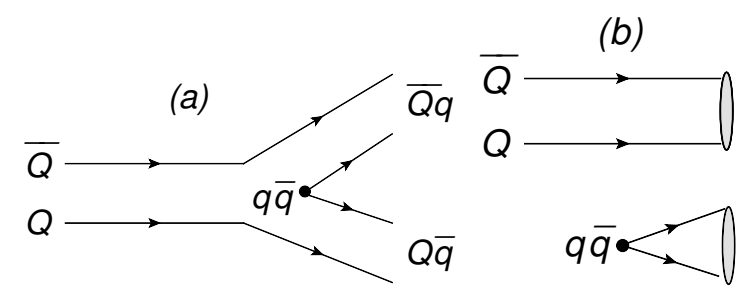

FIG. 4. (a) shows a connected decay diagram where the decay can be suppressed. (b) is an example of a disconnected diagram where the decay is not suppressed..

spin. $S_{q \bar{q}}$ is the total spin of the created pair. In the non-relativistic limit, $S_{q \bar{q}}=1$. For non-connected diagrams (Figure 4(b)), he found no such general rules, so the vanishing of the decays occur to the extent that the non-connected diagrams are not important (OZI suppression).

As an example of this, consider $A$ to be the $\pi_{1}$ hybrid. It has $C_{A}^{0}=+1, P_{A}=-1$ and $S_{A}=+1$, thus the lefthand side of equation 10 is -1 . The right-hand side is $(-1)^{3}=-1$. The decay to pairs of mesons with the same internal angular momentum is suppressed to the extent that the disconnected diagram in Figure $4(\mathrm{~b})$ is not important. In a later study, Close and Dudek 38. found that some of these decays could be large because the $\pi$ and $\rho$ wave functions were not the same.

While it has been historically difficult to compute decays on the lattice, a first study of the decay of the $\pi_{1}$ hybrid has been carried out by McNeile 27, 39. In order to do this, they used a technique where they put a given decay channel at roughly the same energy as the decaying state. Thus, the decay is just allowed and conserves energy in a two-point function. In this way, they are able to extract the ratio of the decay width over the decay momentum, and find

$$
\begin{aligned}
& \Gamma\left(\pi_{1} \rightarrow b_{1} \pi\right) / k=0.66 \pm 0.20 \\
& \Gamma\left(\pi_{1} \rightarrow f_{1} \pi\right) / k=0.15 \pm 0.10
\end{aligned}
$$

which they note corresponds to a total decay width larger than $0.4 \mathrm{GeV}$ for the $\pi_{1}$. As a check of their procedure, they carry out a similar calculation for $b_{1} \rightarrow \omega \pi$ where they obtain $\Gamma / k \sim 0.8$, which leads to $\Gamma\left(b_{1} \rightarrow \omega \pi\right) \sim$ $0.22 \mathrm{GeV}$. This is about a factor of 1.6 larger than the experimental width.

Burns and Close [40] examined these lattice decay results and made comparisons to what had been found in flux-tube model calculations. In Table IX] are shown their comparison between flux-tube calculations for the width of the $\pi_{1}$ and the decay width from the lattice. They note that in the work of McNeile 27, an assumption was made that $\Gamma / k$ does not vary with the quark mass, and the resulting linear extrapolation leads to the large width in Table IX. They argue that the flux-tube model has been tested over a large range of $k$, where it accurately predicts the decays of mesons and baryons. Quoting them, "The successful phenomenology of this and a wide range 
of other conventional meson decays relies on momentumdependent form factors arising from the overlap of hadron wave functions. The need for such form factors is rather general, empirically supported as exclusive hadron decay widths do not show unrestricted growth with phase space." Based on this, they carried out a comparison of the transition amplitudes computed for $k=0$ (the lattice case). They found excellent agreement between the lattice and the flux-tube calculations. Thus, their concern that the extrapolation may be overestimating decay widths may be valid.

\begin{tabular}{lccc}
\hline \hline & IKP & IKP & Lattice \\
& {$[17$} & {$[3]$} & {$[3]$} \\
& $1.9 \mathrm{GeV}$ & $2.0 \mathrm{GeV}$ & $2.0 \mathrm{GeV}$ \\
\hline$\Gamma\left(\pi_{1} \rightarrow b_{1} \pi\right)_{S}$ & 100 & 70 & $400 \pm 120$ \\
$\Gamma\left(\pi_{1} \rightarrow b_{1} \pi\right)_{D}$ & 30 & 30 & \\
$\Gamma\left(\pi_{1} \rightarrow f_{1} \pi\right)_{S}$ & 30 & 20 & $90 \pm 60$ \\
$\Gamma\left(\pi_{1} \rightarrow f_{1} \pi\right)_{D}$ & 20 & 25 & \\
\hline \hline
\end{tabular}

TABLE IX. Decay widths as computed in the flux-tube model (IKP) compared to the lattice calculations. (Table reproduced from reference [40].)

While the model calculations provide a good guide in looking for hybrids, there are often symmetries that can suppress or enhance certain decays. Chung and Klempt [42] noted one of these for decays of a $J^{P C}=1^{-+}$ state into $\eta \pi$ where the $\eta$ and $\pi$ have relative angular momentum of $L=1$. In particular, in the limit where the $\eta$ is an $\mathrm{SU}(3)$ octet, the $\eta \pi$ in a $p$-wave must be in an antisymmetric wave function. In order to couple this to an octet (hybrid) meson, the hybrid must also be antisymmetric. This implies it must be a member of the $8_{2}$ octet. However, the SU(3) Clebsch-Gordan coefficient for $8_{2} \rightarrow \eta_{8} \pi$ is zero. Thus, the decay is forbidden.

However, by similar arguments, they showed that it can couple to the $10 \oplus \overline{10}$ representation of $\mathrm{SU}(3)$. A representation that contains multiquark $(q q \bar{q} \bar{q})$ objects (see Section IIF). Similarly, for the singlet $\left(\eta^{\prime}\right)$ in a $p$ wave, the coupling to an octet is not suppressed.

To the extent that the $\eta$ is octet and the $\eta^{\prime}$ is singlet, a $1^{-+}$state that decays to $\eta \pi$ and not $\eta^{\prime} \pi$ cannot be a hybrid, while one that decays to $\eta^{\prime} \pi$ and not $\eta \pi$ is a candidate for a $1^{-+}$hybrid meson. Our current understanding of the pseudoscalar mixing angle is that it is between $-10^{\circ}$ and $-20^{\circ}$ [1, thus the assumption on the nature of the $\eta$ and $\eta^{\prime}$ is not far off. However, as far as we know, the pseudoscalar mesons are the only nonet that is close to pure $\mathrm{SU}(3)$ states, all others tend to be close to ideal mixing. A case where the higher mass state is nearly pure $s \bar{s}$. Thus, this suppression would not be expected for decays to higher mass nonets.

\section{F. Multiquark states}

As noted in Section I. exotic quantum numbers can arise from other quark-gluon systems as well. While it is possible for glueballs to have exotic quantum numbers, the masses are expected to be above $3 \mathrm{GeV}$ [3]. Another configuration are multiquark states $(q q \bar{q} \bar{q})$ consisting of two quarks and two antiquarks. A short review of this topic can be found in Ref. [44, and a nice description of how these states are built in the quark model can be found in Ref. 45].

Following Ref. [46, the SU(3) multiplets of these states can be obtained by considering $q q$ and $\bar{q} \bar{q}$ combinations. The former can transform as either $\overline{3}$ or 6 under SU(3), while the latter can transform as 3 and $\overline{6}$. Thus, multiplets can be built up as

$$
\begin{array}{rlccc}
\overline{3} \otimes 3 & = & 1 \oplus 8 & = & 9 \\
6 \otimes \overline{6} & = & 1 \oplus 8 \oplus 27 & = & 36 \\
6 \otimes 3 \oplus \overline{3} \otimes \overline{6} & = & 8 \oplus 10 \oplus 8 \oplus \overline{10} & & 18 \oplus \overline{18}
\end{array}
$$

The $J^{P}$ of these multiquark states can be obtained by initially combining all the quarks in an S-wave. This yields $J^{P}$ values of $0^{+}, 1^{+}$and $2^{+}$, which can be combined with the fact that the overall wave functions must be antisymmetric to associate $\mathrm{SU}(3)$ multiplets with $J^{P}$.

$$
\begin{aligned}
& J^{P}=2^{+}: 9,36 \\
& J^{P}=1^{+}: 9,18,18, \overline{18}, \overline{18}, 36 \\
& J^{P}=0^{+}: 9,9,36,36
\end{aligned}
$$

Jaffe considered these multiquark states in terms of the bag model [46, 47, where he found a nonet of $J^{P}=0^{+}$ states to be the lightest with a mass around $1 \mathrm{GeV}$. This cryptoexotic nonet is interesting in that the $\rho$ - and $\omega$-like states have an $s \bar{s}$ pair combined with the lighter quarks.

$$
\begin{aligned}
& \omega \frac{1}{\sqrt{2}}(u \bar{u}+d \bar{d})(s \bar{s}) \\
& \rho^{+} u \bar{d}(s \bar{s}) \\
& \rho^{0} \frac{1}{\sqrt{2}}(u \bar{u}-d \bar{d})(s \bar{s}) \\
& \rho^{-} d \bar{u}(s \bar{s})
\end{aligned}
$$

The $K$-like states have a single strange quark,

$$
\begin{array}{cc}
K^{+} & u \bar{s} d \bar{d} \\
K^{0} & d \bar{s} u \bar{u} \\
\bar{K}^{0} & s \bar{u} d \bar{d} \\
K^{-} & s \bar{d} u \bar{u}
\end{array}
$$

while the $\phi$-like state has no strange quarks,

$$
\phi u \bar{u} d \bar{d} \text {. }
$$

This yields the so-called inverted nonet, where the masshierarchy is reversed relative to the $q \bar{q}$ states. This nonet is often associated with the low-mass states $f_{0}(600)(\sigma)$, $K_{0}^{*}(800)(\kappa), a_{0}(980)$ and the $f_{0}(980)$. Jaffe also noted that whenever the expected mass of a multiquark state was above that of a simple meson-meson threshold to which the state could couple, the decays would be "superallowed", and the width of the state would be very large. 
Because of these super-allowed decays, Jaffe 48 noted that the states would not exist.

Orbital excitations of the multiquark systems were examined in reference 49. Additional symmetrization rules beyond the simple $q \bar{q}$ system apply for these, but they found that the addition of one unit of angular momentum could produce both $J^{P C}=1^{-+}$and $0^{--}$states as members of an $18 \oplus \overline{18} \mathrm{SU}(3)$ multiplet with masses around $1.7 \mathrm{GeV}$. There are two isovector states in an 18 , one as part of an octet and the second as part of a decuplet. The multiquark representation can be represented In a meson-meson-like by recoupling the colors and spins to the new basis. Doing this, the two isovector states look like a $\pi$ combined with either and $\eta$ or an $\eta^{\prime}$. While the mixing between the $\eta$ and $\eta^{\prime}$ components is not known, it is likely that both states would have some hidden $s \bar{s}$ component.

General and colleagues [50] looked at multiquark states in the framework of molecular resonances using their coulomb gauge formalism. In this framework, they computed the spectrum of the lightest states and find several states with masses below $1.5 \mathrm{GeV}$. In the isovector sector, they find the lightest state to be a $J^{P C}=1^{-+}$ state $(m=1.32 \mathrm{GeV})$, with a somewhat heavier $0^{--}$ state $(m=1.36 \mathrm{GeV})$, and then a second $1^{-+}$state $(m=1.42 \mathrm{GeV})$. In the isoscalar channel, they find a single $0^{--}$state and in the isotensor (isospin two) channel, they predict an additional $0^{--}$state. Between 1.5 and $2 \mathrm{GeV}$, they predict two additional $1^{-+}$states in each of the three isospin channels.

QSSR techniques have also been used to look for both isovector [51 and isoscalar 52 $J^{P C}=1^{-+}$multiquark states. As with the earlier work, they find that the exoticquantum number multiquark states are in the $(\overline{3} \otimes \overline{6}) \oplus$ $(3 \otimes 6)$ flavor representations. In their calculations, the decuplet $\pi_{1}$ state (with no $s \bar{s}$ pair) has a mass of about $1.6 \mathrm{GeV}$, while the octet $\pi_{1}$ state (with $s \bar{s}$ ) has a mass of about $2 \mathrm{GeV}$. For these states, they suggest decays of the form $J^{P}=0^{+}, J^{P}=1^{-}\left(f_{0} \rho\right), J^{P}=1^{+}, J^{P}=0^{-}$ $\left(b_{1} \pi\right)$ and $J^{P}=1^{-}, J^{P}=1^{+}\left(\omega b_{1}\right)$. For the isoscalar masses, both the octet and decuplet member contain an $s \bar{s}$ pair. They find a single state with a mass between 1.8 and $2.1 \mathrm{GeV}$. For decays, their calculations favor decays of the form $K \bar{K}, \eta \eta, \eta \eta^{\prime}$ and $\eta^{\prime} \eta^{\prime}$. They also

$$
\begin{aligned}
& Z_{e q}=Z_{L}+Z_{C} \\
& Z_{e q}=j \omega L+\frac{1}{j \omega C} \\
& Z_{e q}=\frac{1-\omega^{2} L C}{j \omega C}
\end{aligned}
$$

suggest several decays that are forbidden by isospin conservation.

Lattice calculations for multiquark states are somewhat sparse, largely due to the challenge of the number of quarks. Studies have been made to try to determine if the low-mass scalars have multiquark nature. A calculation in the quenched approximation was made with pion mass as small as $180 \mathrm{MeV}$ identified the $f_{0}(600)$ as a multiquark state [53. A later quenched calculation with heavier pion masses $(344-576 \mathrm{MeV})$ found no indication of the $f_{0}(600)$ [54, but the authors note that their pion mass is too heavy for this to be conclusive. A recent dynamical calculation [55] with somewhat heavier pion mass shows good agreement with Ref. [53], and while the authors could not exclude the states are lattice artifacts, their results suggest that the $f_{0}(600)$ and $K_{0}^{*}(800)$ have a multiquark nature. Finally, a recent dynamical calculation of the entire isovector meson spectrum shows no multiquark states [29. However, the authors note that the correct operators were probably not included in their analysis, so the fact that these states are missing from their analysis should not be taken as conclusive. Other lattice calculations explicitly looking for exotic-quantumnumber multiquark states do not appear to have been performed.

If exotic-quantum number multiquark states exist, the favoured quantum numbers are $1^{-+}$and $0^{--}$. The latter being a $J^{P C}$ not predicted for hybrid mesons. There may also be hidden $s \bar{s}$ components in the multiquark multiplets that would distort their mass hierarchy relative to hybrid nonets. However, for most of these multiquark states, their decays will be super-allowed. In their recent review, Klempt and Zaitsev [3] argue that $(q q)(\bar{q} \bar{q})$ systems will not bind without additional $q \bar{q}$ forces, and feel that it is unlikely that these multiquark states exist. In reviewing the information on these states, we concur with their assessment for the exotic-quantum-number states.

\section{EXPERIMENTAL RESULTS}

\section{A. Production processes}

Data on exotic-quantum-number mesons have come from both diffractive production using incident pion beams and from antiproton annihilation on protons and neutrons. Diffractive production is schematically shown in Figure 5. A pion beam is incident on a proton (or nuclear) target, which recoils after exchanging something in the $t$-channel. The process can be written down in the reflectivity basis [56] in which the production factorizes into two non-interfering amplitudes - positive reflectivity $(\epsilon=+)$ and negative reflectivity $(\epsilon=-)$. The absolute value of the spin projection along the $z$-axis is $M$, and is taken to be either 0 or 1 (it is usually assumed that contributions from $M$ larger than 1 are small and can be ignored [57]). It can be shown in this process that naturality of the exchanged particle can be determined by $\epsilon$. Natural parity exchange (n.p.e.) corresponds to $J^{P_{\mathrm{S}}}$ of $0^{+}, 1^{-}, 2^{+}, \cdots$, while unnatural parity exchange (u.p.e.) corresponds to $J^{P}$ of $0^{-}, 1^{+}, 2^{-}, \cdots$.

For a state which is observed in more than one decay mode, one would expect that the production mechanism $\left(M^{\epsilon}\right)$ would be the same for all decay modes. If not, this could be indicative of more than one state being observed, or possible problems in the analysis that are 


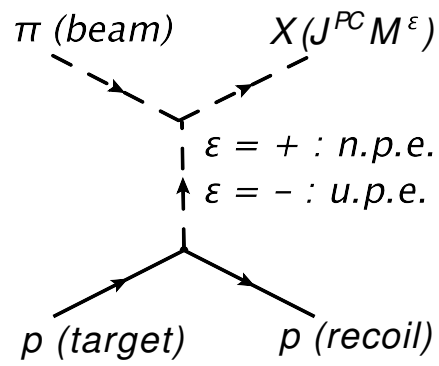

FIG. 5. The diffractive production process showing an incident pion ( $\pi$ beam) incident on a proton ( $p$ target) where the exchange has z-component on angular momentum $M$ and reflectivity $\epsilon$. The final state consists of a proton ( $p$ recoil) and a state $X$ of given $J^{P C}$ produced by an exchange $M^{\epsilon}$. For positive reflectivity, the $t$-channel is a natural parity exchange (n.p.e.), while for negative reflectivity, it is unnatural parity exchange (u.p.e.). (This diagram was produced using the JaxoDraw package [58.)

not under control.

In antiproton-nucleon annihilation, there are a number of differences between various annihilation processes. For the case of $\bar{p} p$, the initial state is a mixture of isospin $I=0$ and $I=1$. For $\bar{p} n$ annihilation, the initial state is pure $I=1$. For annihilation at rest on protons, the initial state is dominated by atomic S-waves. In particular, ${ }^{1} S_{0}$ and ${ }^{3} S_{1}$ atomic states, which have $J^{P C}=0^{-+}$and $1^{--}$respectively (with a small admixture of $P$ states). For annihilation in flight, the number of initial states is much larger and it may no longer make sense to try and parametrize the initial system in terms of atomic states.

The combination of initial isospin and final state particles may lead to additional selection rules that restrict the allowed initial states. In the case of $\bar{p} p \rightarrow \eta \pi^{0} \pi^{0}$, the annihilation is dominated by ${ }^{1} S_{0}$ initial states $\left(J^{P C}=\right.$ $\left.0^{-+}\right)$. For the case of $\bar{p} n \rightarrow \eta \pi^{0} \pi^{-}$, quantum numbers restrict this annihilation to occur from the ${ }^{3} S_{1}$ initial states $\left(J^{P C}=1^{--}\right)$. In addition, the neutron is bound in deuterium, where the Fermi motion introduces substantial p-wave annihilation. Thus, one may see quite different final states from the two apparently similar reactions.

\section{B. The $\pi_{1}(1400)$}

The first reported observation of an exotic quantum number state came from the GAMS experiment which used a $40 \mathrm{GeV} / \mathrm{c} \pi^{-}$to study the reaction $\pi^{-} p \rightarrow p \eta \pi^{-}$. They reported a $J^{P C}=1^{-+}$state in the $\eta \pi^{-}$system which they called the $M(1405)$ 59. The $M(1405)$ had a mass of $1.405 \pm 0.020 \mathrm{GeV}$ and a width of $0.18 \pm 0.02 \mathrm{GeV}$. Interestingly, an earlier search in the $\eta \pi^{0}$ channel found no evidence of an exotic state 60. At KEK, results were reported on studies using a $6.3 \mathrm{GeV} / \mathrm{c} \pi^{-}$beam where they observed a $1^{-+}$state in the $\eta \pi^{-}$system with a mass of $1.3431 \pm 0.0046 \mathrm{GeV}$ and a width of
$0.1432 \pm 0.0125 \mathrm{GeV} 61$. However, there was concern that this may have been leakage from the $a_{2}(1320)$.

The VES collaboration reported intensity in the $1^{-+}$ $\eta \pi^{-}$wave as well as rapid phase motion between the $a_{2}$ and the exotic wave 62 (see Figure 6). The exotic wave was present in the $M^{\epsilon}=1^{+}$(natural parity) exchange, but not in the $0^{-}$and $1^{-}$(unnatural parity) exchange. They could fit the observed $J^{P C}=1^{-+}$intensity and the phase motion with respect to the $a_{2}(1320)$ using a Breit-Wigner distribution (mass of $1.316 \pm 0.012 \mathrm{GeV}$ and width of $0.287 \pm 0.025 \mathrm{GeV}$ ). However, they stopped short of claiming an exotic resonance, as they could not unambiguously establish the nature of the exotic wave 63. In a later analysis of the $\eta \pi^{0}$ system, they claim that the peak near $1.4 \mathrm{GeV}$ can be understood without requiring an exotic quantum number meson 64].

The E852 collaboration used $18 \mathrm{GeV} / \mathrm{c} \pi^{-}$beams to study the reaction $\pi^{-} p \rightarrow p \eta \pi^{-}$. They reported the observation of a $1^{-+}$state in the $\eta \pi^{-}$system [65]. E852 found this state only produced in natural parity exchange $\left(M^{\epsilon}=1^{+}\right)$. They measured a mass of $1.37 \pm$ $0.016_{-0.030}^{+0.050} \mathrm{GeV}$ and a width of $0.385 \pm 0.040_{-0.105}^{+0.065} \mathrm{GeV}$. While the observed exotic signal was only a few percent of the dominant $a_{2}(1320)$ strength, they noted that its interference with the $a_{2}$ provided clear evidence of this state. When their intensity and phase-difference plots were compared with those from VES 62, they were identical. These plots (from E852) are reproduced in Figure 7 .

Due to disagreements over the interpretation of the $1^{-+}$signal, the E852 collaboration split into two groups. The majority of the collaboration published the resonance interpretation, $\pi_{1}(1400)$ 65, while a subset of the collaboration did not sign the paper. As this latter group, centered at Indiana University, continued to analyze data collected by E852, we will refer to their publications as E852-IU to try an carefully distinguish the work of the two groups.

The exotic $\pi_{1}$ state was confirmed by the Crystal Barrel Experiment which studied antiproton-neutron annihilation at rest in the reaction $\bar{p} n \rightarrow \eta \pi^{-} \pi^{0}$ [66]. The Dalitz plot for this final state is shown in Figure 8 where bands for the $a_{2}(1320)$ and $\rho(770)$ are clearly seen. They reported a $1^{-+}$state with a mass of $1.40 \pm 0.020 \pm$ $0.020 \mathrm{GeV}$ and a width of $0.310 \pm 0.050_{-0.030}^{+0.050} \mathrm{GeV}$. While the signal is not obvious in the Dalitz plot, if one compares the difference between a fit to the data without and with the $\pi_{1}(1400)$, a clear discrepancy is seen when the $\pi_{1}(1400)$ is not included (see Figure 9). While the $\pi_{1}(1400)$ was only a small fraction of the $a_{2}(1320)$ in the E852 measurement 65], Crystal Barrel observed the two states produced with comparable strength.

Crystal Barrel also studied the reaction $\bar{p} p \rightarrow$ $\eta \pi^{0} \pi^{0}$ [67. Here, a weak signal was observed for the $\pi_{1}(1400)$ (relative to the $a_{2}(1320)$ ) with a mass of $1.360 \pm$ $0.025 \mathrm{GeV}$ and a width of $0.220 \pm 0.090 \mathrm{GeV}$. In $I=0 \bar{p} p$ annihilations, the $a_{2}(1320)$ is produced strongly from the ${ }^{1} S_{0}$ atomic state. However, $\bar{p} n$ is isospin 1 and ${ }^{1} S_{0}$ state 


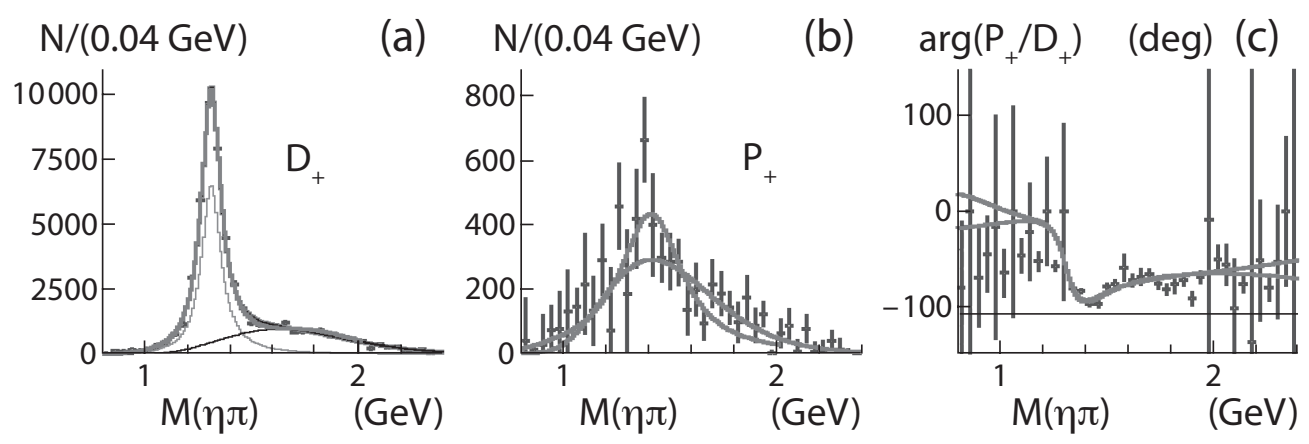

FIG. 6. The results of a partial-wave analysis of the $\eta \pi^{-}$final state from VES. (a) shows the intensity in the $2^{++}$partial wave, (b) shows the intensity in the $1^{-+}$partial wave and (c) shows the relative phase between the waves. (Figure reproduced from reference 64.)

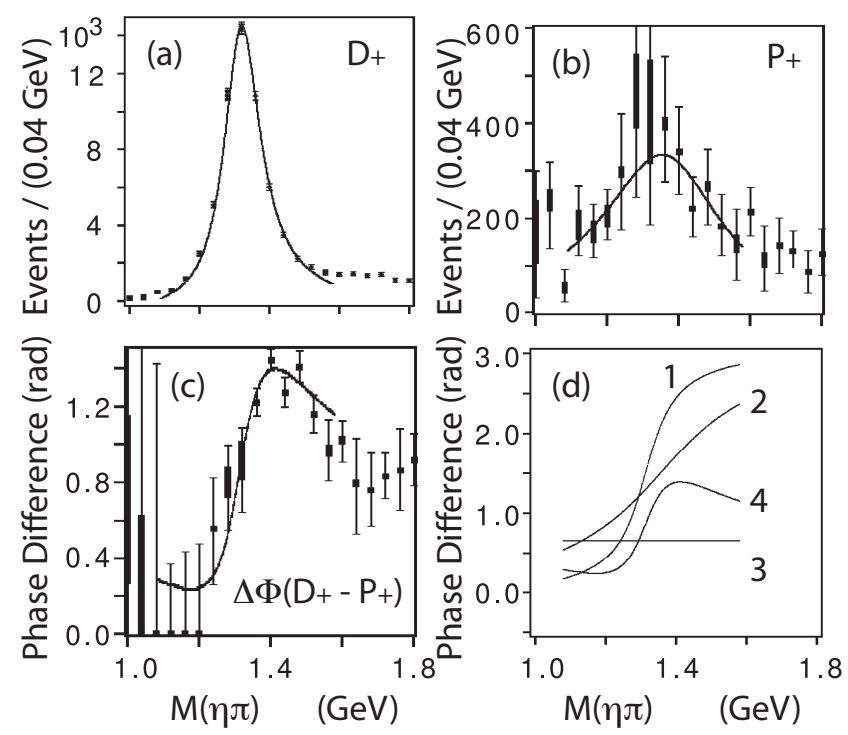

FIG. 7. The $\pi_{1}(1400)$ as observed in the E852 experiment 65 . (a) shows the intensity of the $J^{P C}=2^{++}$partial wave as a function of $\eta \pi$ mass. The strong signal is the $a_{2}(1320)$. (b) shows the intensity of the $1^{-+}$wave as a function of mass, while (c) shows the phase difference between the $2^{++}$and $1^{-+}$partial waves. In (d) are shown the phases associated with (1) the $a_{2}(1320),(2)$ the $\pi_{1}(1400)$, (3) the assumed flat background phase and (4), the difference between the (1) and (2). (This figure is reproduced from reference [65].)

is forbidden. Thus, the strong $a_{2}$ production from $\bar{p} p$ is suppressed in $\bar{p} d$ annihilations - making the $\pi_{1}(1400)$ production appear enhanced relative to the $a_{2}(1320)$ in the latter reaction.

An analysis by the E852-IU group of data for the reaction $\pi^{-} p \rightarrow n \eta \pi^{0}$ found evidence for the exotic $1^{-+}$ partial wave, but were unable to describe it as a BreitWigner-like $\pi_{1}(1400) \eta \pi^{0}$ resonance 68. However, a later analysis by the E852 collaboration of the same final state and data confirmed their earlier observation of the $\pi_{1}(1400) 69$. E852 found a mass of $1.257 \pm 0.020 \pm$

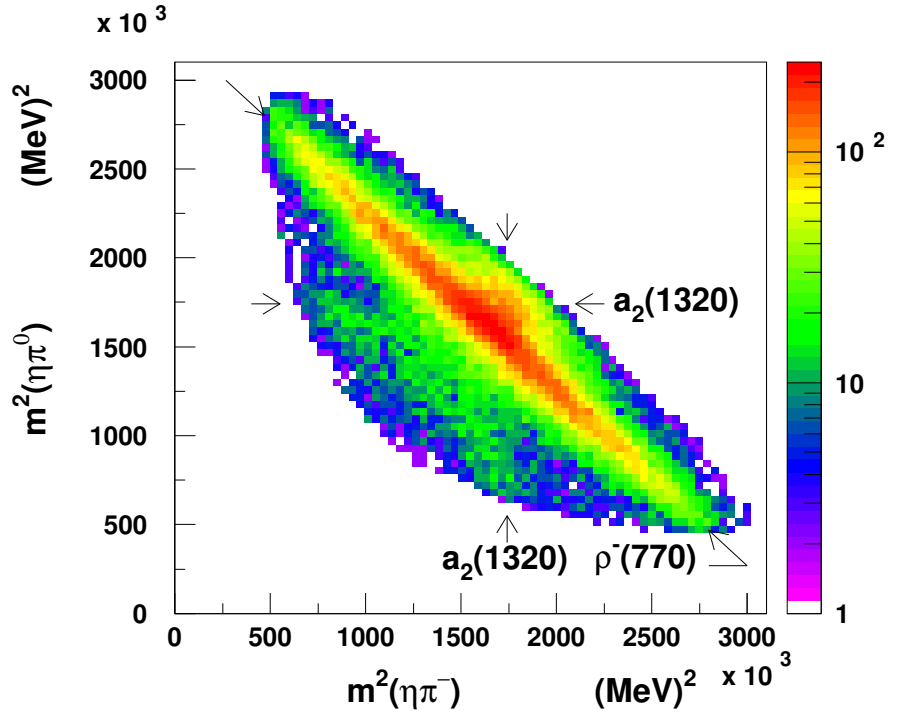

FIG. 8. (Color on line.) The Dalitz plot of $m^{2}\left(\eta \pi^{0}\right)$ versus $m^{2}\left(\eta \pi^{-}\right)$for the reaction $\bar{p} n \rightarrow \eta \pi^{-} \pi^{0}$ from the Crystal Barrel experiment 66. The bands for the $a_{2}(1320)$ are clearly seen in both $\eta \pi^{0}$ and $\eta \pi^{-}$, while the $\rho(770)$ is seen in the $\pi^{0} \pi^{-}$invariant mass.

$0.025 \mathrm{GeV}$ and a width of $0.354 \pm 0.064 \pm 0.058 \mathrm{GeV}$ with the $\pi_{1}(1400)$ produced via natural parity exchange $\left(M^{\epsilon}=1^{+}\right)$. Much of the discrepancy between these two works arise from the treatment of backgrounds. The E852 collaboration consider no background phase, and attribute all phase motion to resonances. The E852-IU group allow for non-resonant interactions in the exotic channel, these background processes are sufficient to explain the observed phase motion.

The $\pi_{1}(1400)$ was also reported in $\bar{p} p$ annihilation into four-pion final states by both Obelix [70 and Crystal Barrel [71] (conference proceedings only). They both observed the $\pi_{1}(1400)$ decaying to $\rho \pi$ final states, however there is some concern about the production mechanism. The $\eta \pi$ signal arises from annihilation from p-wave initial state, while the signal in $\rho \pi$ come from the ${ }^{1} S_{0}$ initial 

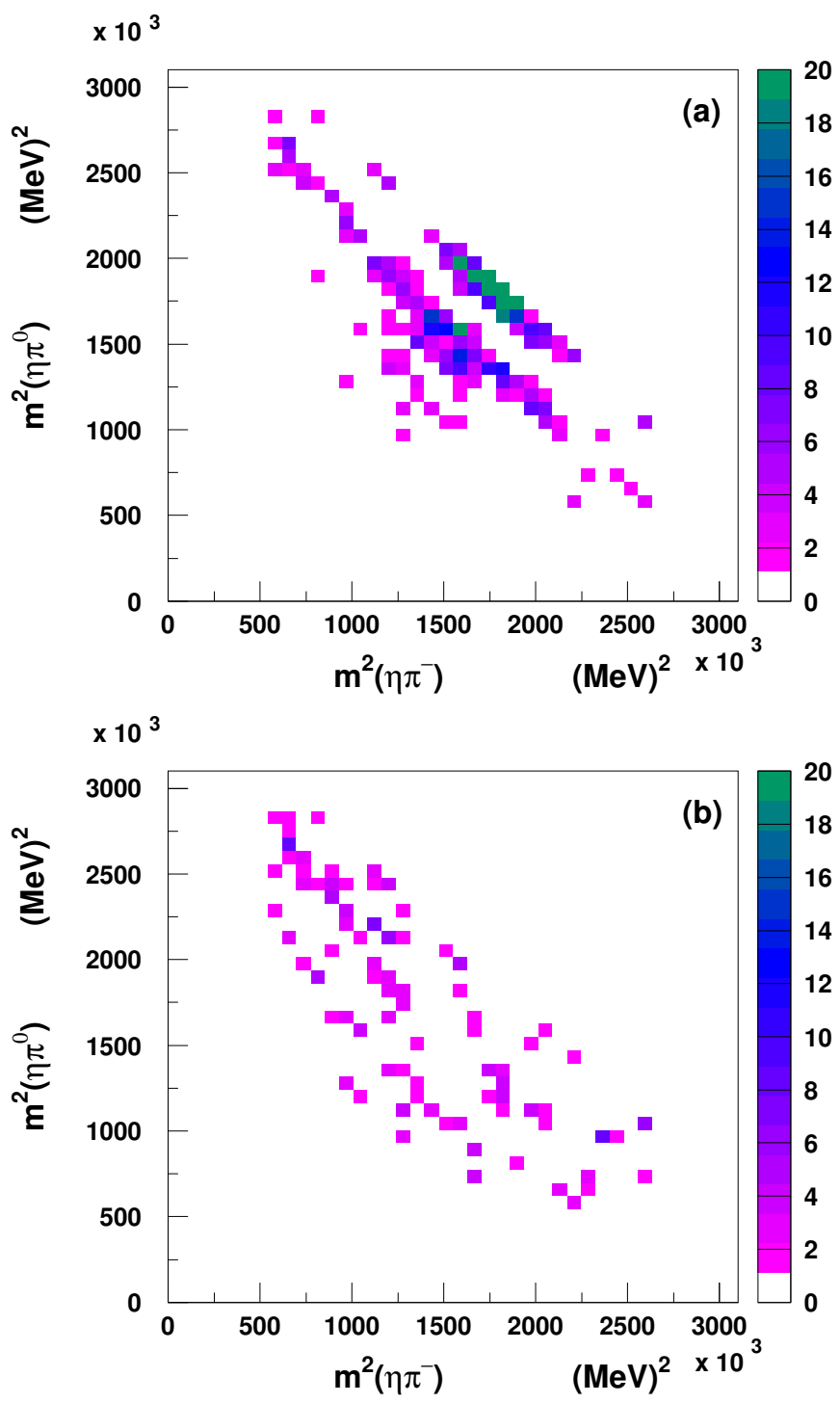

FIG. 9. (Color on line.) The difference between the fit and the data in the Dalitz plot of $m^{2}\left(\eta \pi^{0}\right.$ versus $\eta \pi^{-}$for the reaction $\bar{p} n \rightarrow \eta \pi^{-} \pi^{0}$ from the Crystal Barrel experiment [66. (a) Does not include the $\pi_{1}(1400)$ while (b) does include the $\pi_{1}(1400)$. There are clear systematic discrepancies present in (a) that are not present when the $\pi_{1}(1400)$ is included.

state. Thus, it is unlikely that the exotic state seen in $\eta \pi$ and that seen in $\rho \pi$ are the same. The origin of these may not be due to an exotic resonance, but rather some re-scattering mechanism that has not been properly accounted for.

Interpretation of the $\pi_{1}(1400)$ has been problematic. Its mass is lower than most predicted values from models, and its observation in only a single decay mode $(\eta \pi)$ is not consistent with models of hybrid decays. Donnachie and Page showed that the $\pi_{1}(1400)$ could be an artifact of the production dynamics. They demonstrated that is possible to understand the $\pi_{1}(1400)$ peak as a consequence of the $\pi_{1}(1600)$ (see Section III C) interfer- ing with a non-resonant Deck-type background with an appropriate relative phase 72. Zhang [73] considered a molecular picture where the $\pi_{1}(1400)$ was an $\eta(1295) \pi$ molecule. However, the predicted decays were inconsistent with the observations of the $\pi_{1}(1400)$.

Szczepaniak 74] considered a model in which $t$-channel forces could give rise to a background amplitude which could be responsible for the observed $\pi_{1}(1400)$. In his model, meson-meson interactions which respected chiral symmetry were used to construct the $\eta \pi p$-wave interaction much like the $\pi \pi s$-wave interaction gives rise to the $\sigma$ meson. They claimed that the $\pi_{1}(1400)$ was not a QCD bound state, but rather dynamically generated by meson exchange forces.

Close and Lipkin noted that because the SU(3) multiplets to which a hybrid and a multiquark state belong are different, that the $\eta \pi$ and $\eta^{\prime} \pi$ decays might be a good way to distinguish them. They found that for a multiquark state, the $\eta \pi$ decay should be larger than $\eta^{\prime} \pi$, while the reverse is true for a hybrid meson [41]. A similar observation was made by Chung 42 who noted that in the limit of the $\eta$ being a pure octet state, that the decay of an octet $1^{-+}$state to an $\eta \pi p$-wave is forbidden. Such a decay can only come from a decuplet state. Given that the pseudoscalar mixing angle for the $\eta$ and $\eta^{\prime}$ are close to this assumption, they argue that the $\pi_{1}(1400)$ is $q q \bar{q} \bar{q}$ in nature.

While the interpretation of the $\pi_{1}(1400)$ is not clear, most analyses agree that there is intensity in the $1^{-+}$ wave near this mass. A summary of all reported masses and widths for the $\pi_{1}(1400)$ are given in Table $\mathrm{X}$. All are reasonably consistent, and even the null observations of VES and E852-IU all concur that there is strength near $1.4 \mathrm{GeV}$ in the $J^{P C}$ exotic wave. However The E852 and VES results can be explained as either nonresonant background [74, or non-resonant deck amplitudes [72. An other possibility is the opening of mesonmeson thresholds, such as $f_{1}(1285) \pi$. Unfortunately, no comparisons of these hypothesis have been made with the $\bar{p} N$ data (owing to the lack of general availability of the data sets), so it is not possible to conclude that they would also explain those data. However, in our minds, we believe that the evidence favors a non-resonant interpretation of the exotic $1^{-+}$signal and that the $\pi_{1}(1400)$ does not exist.

\section{The $\pi_{1}(1600)$}

While the low mass, and single observed decay mode, of the $\pi_{1}(1400)$ have presented some problems in understanding its nature, a second $J^{P C}=1^{-+}$state is less problematic. The $\pi_{1}(1600)$ has been observed in diffractive production using incident $\pi^{-}$beams where its mass and width have been reasonably stable over several experiments and the decay modes. It may also have been observed in $\bar{p} p$ annihilation. Positive results have been reported from VES, E852, COMPASS and others. These 


\begin{tabular}{|c|c|c|c|c|}
\hline Mode & Mass $(\mathrm{GeV})$ & Width $(\mathrm{GeV})$ & Experiment & Reference \\
\hline$\eta \pi^{-}$ & $1.405 \pm 0.020$ & $0.18 \pm 0.02$ & GAMS & 59 \\
\hline$\eta \pi^{-}$ & $1.343 \pm 0.0046$ & $0.1432 \pm 0.0125$ & KEK & 61 \\
\hline$\eta \pi^{-}$ & $1.37 \pm 0.016$ & $0.385 \pm 0.040$ & $\mathrm{E} 852$ & 65 \\
\hline$\eta \pi^{0}$ & $1.257 \pm 0.020$ & $0.354 \pm 0.064$ & $\mathrm{E} 852$ & 69 \\
\hline$\eta \pi$ & $1.40 \pm 0.020$ & $0.310 \pm 0.050$ & CBAR & 66 \\
\hline$\eta \pi^{0}$ & $1.36 \pm 0.025$ & $0.220 \pm 0.090$ & CBAR & 67 \\
\hline$\rho \pi$ & $1.384 \pm 0.028$ & $0.378 \pm 0.058$ & Obelix & 70 \\
\hline$\rho \pi$ & $\sim 1.4$ & $\sim 0.4$ & CBAR & 71 \\
\hline$\eta \pi$ & $1.351 \pm 0.030$ & $0.313 \pm 0.040$ & PDG & 1 \\
\hline
\end{tabular}

TABLE X. Reported masses and widths of the $\pi_{1}(1400)$ from the GAMS, KEK, E852, Crystal Barrel (CBAR) and Obelix experiment. Also reported is the 2008 PDG average for the state.

are discussed below in approximate chronological order.

In addition to their study of the $\eta \pi^{-}$system, the VES collaboration also examined the $\eta^{\prime} \pi^{-}$system. Here they observed a $J^{P C}=1^{-+}$partial wave with intensity peaking at a higher mass than the $\pi_{1}(1400)$ 62. However, as with the $\eta \pi^{-}$system, they did not claim the discovery of an exotic-quantum-number resonance. VES later reported a combined study of the $\eta^{\prime} \pi^{-}, f_{1} \pi^{-}$and $\rho^{0} \pi^{-}$ final states [75, and reported a "resonance-like structure" with a mass of $1.62 \pm 0.02 \mathrm{GeV}$ and a width of $0.24 \pm 0.05 \mathrm{GeV}$ decaying into $\rho^{0} \pi^{-}$. They also noted that the wave with $J^{P C}=1^{-+}$dominates in the $\eta^{\prime} \pi^{-}$ final state, peaking near $1.6 \mathrm{GeV}$ and observed a small $1^{-+}$signal in the $f_{1} \pi^{-}$final state.
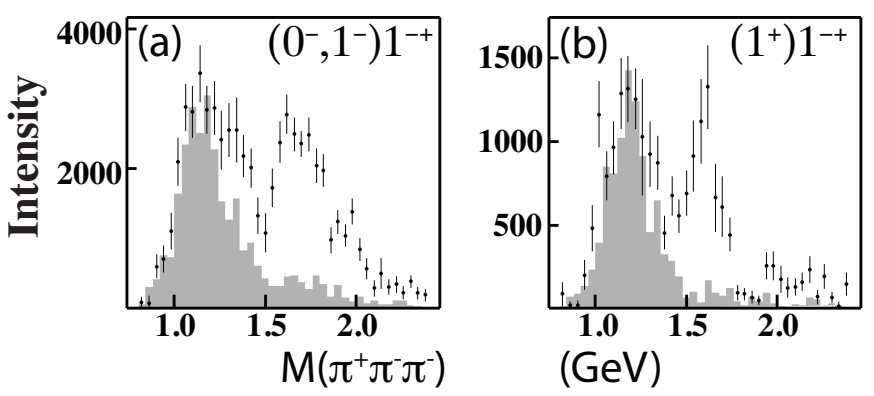

FIG. 10. The production of the $1^{-+}$partial wave as seen in the $\pi^{+} \pi^{-} \pi^{-}$final state by E852. (a) shows the unnatural parity exchange $\left(M^{\epsilon}=0^{-}, 1^{-}\right)$while (b) shows the natural parity exchange $\left(M^{\epsilon}=1^{+}\right)$. (Figure reproduced from reference [76].)

Using an $18 \mathrm{GeV} / \mathrm{c} \pi^{-}$beam incident on a proton target, the E852 collaboration carried out a partial wave analysis of the $\pi^{+} \pi^{-} \pi^{-}$final state $[76$, 77. They saw both the $\rho^{0} \pi^{-}$and $f_{2}(1270) \pi^{-}$intermediate states and observed a $J^{P C}=1^{-+}$state which decayed to $\rho \pi$, the $\pi_{1}(1600)$. The $\pi_{1}(1600)$ was produced in both natural and unnatural parity exchange $\left(M^{\epsilon}=1^{+}\right.$and $M^{\epsilon}=0^{-}$, $\left.1^{-}\right)$with apparent similar strengths in all three exchange mechanisms (see Figure 10). In Ref. 77, they noted that there were issues with the unnatural exchange production. The signal in the $M^{\epsilon}=1^{-}$wave exhibited very strong model dependence and nearly vanished when larger numbers of partial waves were included. The signal in the $M^{\epsilon}=0^{-}$partial wave was stable, but its peak was above $1.7 \mathrm{GeV}$. They noted that the unnatural-parity exchange is expected to die off at higher energies, so their results are not at odds with those of VES (see below), where natural parity exchange dominates. Even in their data, the unnatural parity exchange waves make up a small fraction of the total signal. In unnatural parity exchange, they found no significant waves, which made a study of phase motion of the $1^{-+}$in this sector problematic. Thus, in their analysis, they only considered the natural parity exchange. There, they found the $\pi_{1}(1600)$ to have a mass of $1.593 \pm 0.08_{-0.047}^{+0.029} \mathrm{GeV}$ and a width of $0.168 \pm 0.020_{-0.012}^{+0.150} \mathrm{GeV}$. In Figure 11 are shown the intensity of the $1^{-+}$and $2^{-+}\left(\pi_{2}(1670)\right)$ partial waves as well as their phase difference. The phase difference can be reproduced by two interfering Breit-Wigner distributions and a flat background.
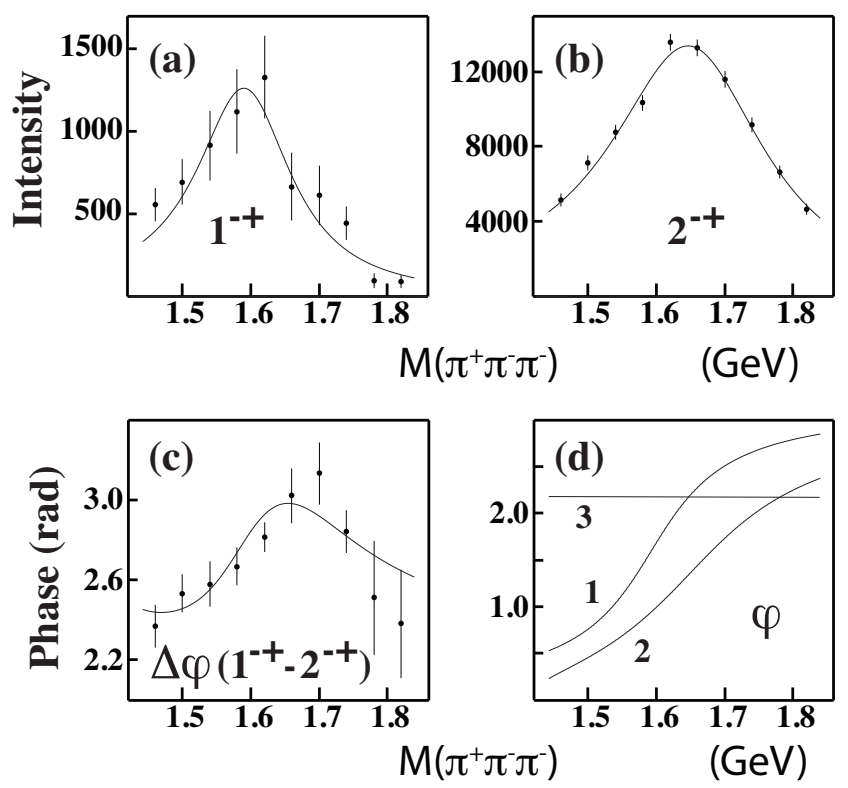

FIG. 11. The results of a PWA to the $\pi^{+} \pi^{-} \pi^{-}$final state from E852. (a) shows the intensity of the $J^{P C}=1^{-+}$wave, (b) shows the $2^{-+}$and (c) shows the phase difference between the two. The solid curves are fits to two interfering BreitWigner distributions. In (d) are shown the phases of the two Breit-Wigner distributions and $(1,2)$ and a flat background phase (3) that combine to make the curve in (c). (Figure reproduced from reference [76].)

VES also reported on the $\omega \pi^{-} \pi^{0}$ final state [78, 79. In a combined analysis of the $\eta^{\prime} \pi^{-}, b_{1} \pi$ and $\rho^{0} \pi^{-}$final states, they reported the $\pi_{1}(1600)$ state with a mass of $1.61 \pm 0.02 \mathrm{GeV}$ and a width of $0.29 \pm 0.03 \mathrm{GeV}$ that was consistent with all three final states. To the extent that they observed these states, they also observed all three final state produced in natural parity exchange $\left(M^{\epsilon}=\right.$ $\left.1^{+}\right)$. They were also able to report relative branching 
ratios for the three final states as given in equation 11 .

$$
b_{1} \pi: \eta^{\prime} \pi: \rho \pi:=1: 1 \pm 0.3: 1.5 \pm 0.5
$$

However, there were some issues with the $\rho \pi$ final state. Rather than limiting the rank of the density matrix as was done in [76, 77, they did not limit it. This allowed for a more general fit that might be less sensitive to acceptance affects. In this model, they did not observe any significant structure in the $1^{-+} \rho \pi$ partial wave above $1.4 \mathrm{GeV}$. However, by looking at how other resonances were produced, they were able to isolate a coherent part of the density matrix from which they found a statistically significant $1^{-+}$partial wave peaking near $1.6 \mathrm{GeV}$. While VES was extremely careful not to claim the existence of the $\rho \pi$ decay of the the $\pi_{1}(1600)$, in the case that it exists, they were able to obtain the rates given in equation 11 .

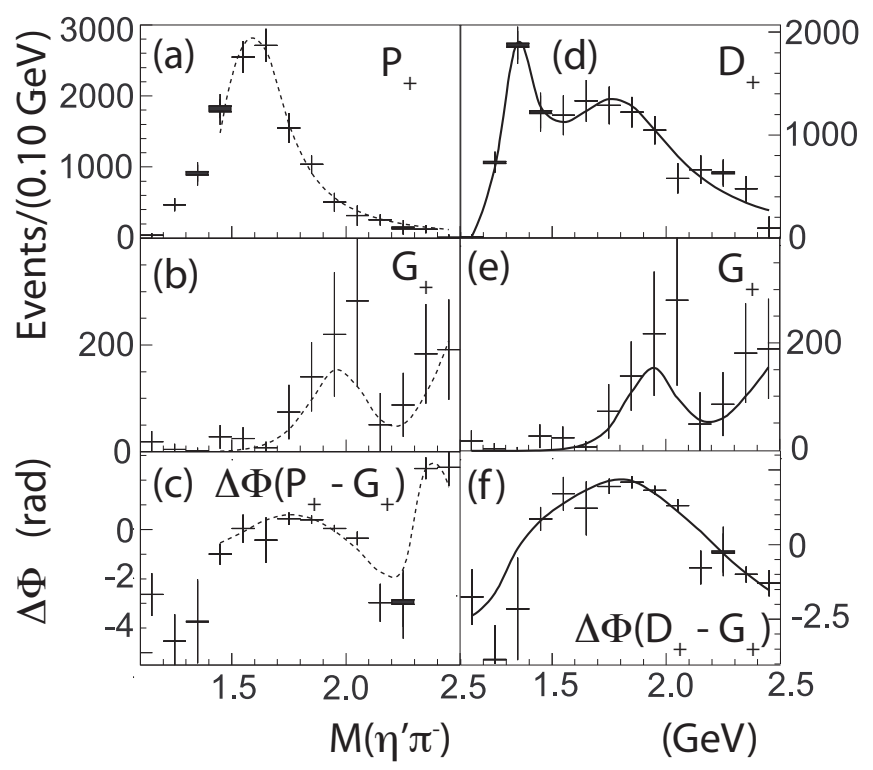

FIG. 12. Results from E852 on the $\eta^{\prime} \pi^{-}$final state. (a) shows the $1^{-+}$partial wave, (b) shows the $4^{++}$partial wave (an $a_{4}$ ) and (c) shows the phase difference between these. (d) shows the $2^{++}$partial wave $\left(a_{2}(1320)\right)$, while (e) shows the $a_{4}$ and (f) is the phase difference. (Figure reproduced from reference 80 .)

In a follow-up analysis, E852 also studied the reaction $\pi^{-} p \rightarrow p \eta^{\prime} \pi^{-}$to examine the $\eta^{\prime} \pi^{-}$final state [80]. They observed, consistent with VES 62, that the dominant signal was the $1^{-+}$exotic wave produced dominantly in the $M^{\epsilon}=1^{+}$channel, implying only natural parity exchange. They found the signal to be consistent with a resonance, the $\pi_{1}(1600)$ and found a mass of $1.597 \pm$ $0.010_{-0.010}^{+0.045} \mathrm{GeV}$ and a width of $0.340 \pm 0.040 \pm 0.050 \mathrm{GeV}$. The results of the E852 PWA are shown in Figure 12 where the $P_{+}$wave is the $1^{-+}$, the $D_{+}$corresponds to the $2^{++} a_{2}$ and the $G_{+}$corresponds to the $4^{++} a_{4}$. Clear phase motion is observed between both the $2^{++}$and $4^{++}$ wave and the $1^{-+}$and the $4^{++}$wave.

An analysis of Crystal Barrel data at rest for the reaction $\bar{p} p \rightarrow \omega \pi^{+} \pi^{-} \pi^{0}$ was carried by some members of the collaboration [81. They reported evidence for the $\pi_{1}(1600)$ decaying to $b_{1} \pi$ from both the ${ }^{1} S_{0}$ and ${ }^{3} S_{1}$ initial states, with the signal being stronger from the former. The total signal including both initial states, as well as decays with 0 and 2 units of angular momentum accounted for less than $10 \%$ of the total reaction channel. The mass and width were found consistent (within large errors) of the PDG value, and only results with the mass and width fixed to the PDG values were reported. Accounting for the large rate of annihilation to $\omega \pi^{+} \pi^{-} \pi^{0}$ of $13 \%$, this would imply that $\bar{p} p \rightarrow \pi_{1}(1600) \pi$ accounts for several percent of all $\bar{p} p$ annihilations.

E852 also looked for the decays of the $\pi_{1}(1600)$ to $b_{1} \pi$ and $f_{1} \pi$. The latter was studied in the reaction $\pi^{-} p \rightarrow p \eta \pi^{+} \pi^{-} \pi^{-}$with the $f_{1}$ being reconstructed in its $\eta \pi^{+} \pi^{-}$decay mode [82]. The $\pi_{1}(1600)$ was seen via interference with both the $1^{++}$and $2^{-+}$partial waves. It was produced via natural parity exchange $\left(M^{\epsilon}=1^{+}\right)$ and found to have a mass of $1.709 \pm 0.024 \pm 0.041 \mathrm{GeV}$ and a width of $0.403 \pm 0.080 \pm 0.115 \mathrm{GeV}$. A second $\pi_{1}$ state was also observed in this reaction (see Section IIID).

The $b_{1} \pi$ final state was studied by looking at the reaction $\pi^{-} p \rightarrow \omega \pi^{-} \pi^{0} p$, with the $b_{1}$ reconstructed in its $\omega \pi$ decay mode 83 . The $\pi_{1}(1600)$ was seen interfering with the $2^{++}$and $4^{++}$partial waves. In $b_{1} \pi$, they measured a mass of $1.664 \pm 0.008 \pm 0.010 \mathrm{GeV}$ and a width of $0.185 \pm 0.025 \pm 0.028 \mathrm{GeV}$ for the $\pi_{1}(1600)$. However, the production mechanism was seen to be a mixture of both natural and unnatural parity exchange, with the unnatural being stronger. As with the $f_{1} \pi$, they also observed a second $\pi_{1}$ state decaying to $b_{1} \pi$ (see Section IIID).

\begin{tabular}{crc}
\hline \hline \multicolumn{2}{c}{ final state production $\left(M^{\epsilon}\right)$} & dominant \\
\hline$\rho \pi$ & $0^{-}, 1^{-}, 1^{+}$ & npe $\sim$ upe \\
$\eta^{\prime} \pi$ & $1^{+}$ & npe \\
$f_{1} \pi$ & $1^{+}$ & npe \\
$b_{1} \pi$ & $0^{-}, 1^{-}, 1^{+}$ & upe $>$npe \\
\hline \hline
\end{tabular}

TABLE XI. The production mechanisms for the $\pi_{1}(1600)$ as seen in the E852 experiment. Also shown is whether the natural parity exchange (npe) or the unnatural parity exchange (upe) is stronger.

The fact that E852 observed the $\pi_{1}(1600)$ produced in different production mechanisms, depending on the final state, is somewhat confusing. A summary of the observed mechanisms is given in Table XI. In order to understand the variations in production, there either needs to be two nearly-degenerate $\pi_{1}(1600) \mathrm{s}$, or there is some unaccounted-for systematic problem in some of the analyses.

The E852-IU group analyzed an E852 data set that was an order of magnitude larger than that used by E852 in Refs. [76, 77]. In this larger data set, they looked at the 


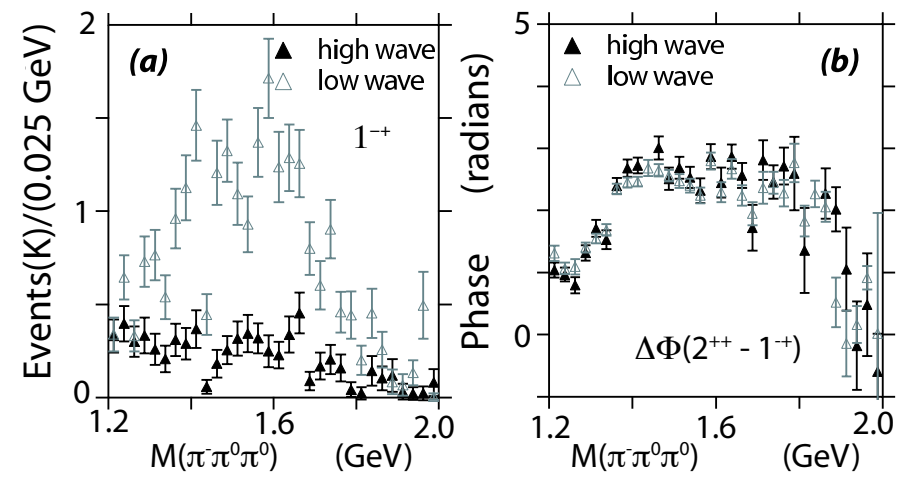

FIG. 13. (Color on line) The PWA solutions for the $1^{-+}$ partial wave for the $\pi^{-} \pi^{0} \pi^{0}$ final state (a) and its interference with the $2^{++}$partial wave (b). See text for an explanation of the labels. (Figure reproduced from reference [85].)
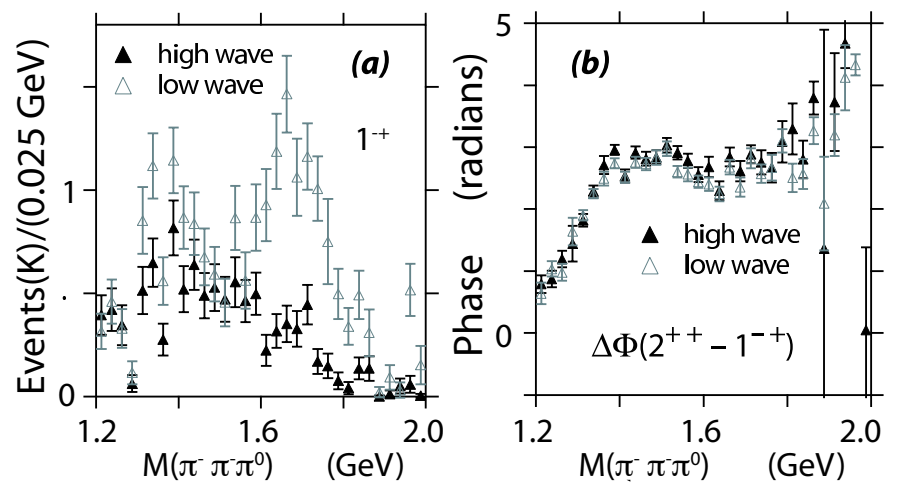

FIG. 14. (Color on line) The PWA solutions for the $1^{-+}$partial wave for the $\pi^{+} \pi^{-} \pi^{-}$final state (a) and its interference with the $2^{++}$partial wave (b). See text for an explanation of the labels. (Figure reproduced from reference [85].)

reactions $\pi^{-} p \rightarrow n \pi^{+} \pi^{-} \pi^{-}$and $\pi^{-} p \rightarrow n \pi^{-} \pi^{0} \pi^{0}$ and carried out a partial wave analysis for both the $\pi^{+} \pi^{-} \pi^{-}$ and the $\pi^{-} \pi^{0} \pi^{0}$ final states. This yielded solutions that were consistent with both final states [85. In this analysis, they carried out a systematic study of which partial waves were important in the fit. When they used the same wave set as in the E852 analysis [76, 77, they found the same solution showing a signal for the $\pi_{1}(1600)$ in both final states. However, when they allowed for more partial waves, they found that the signal for the $\pi_{1}(1600)$ went away. Figure 13 shows these results for the $\pi^{-} \pi^{0} \pi^{0}$ final state, while Figure 14 shows the results for the $\pi^{+} \pi^{-} \pi^{-}$final state. In both figures, the "low wave" solution matches that from E852, while their "high wave" solution shows no intensity for the $\pi_{1}(1600)$ in either channel. An important point is that in both their high-wave and low-wave analyses, the phase difference between the exotic $1^{-+}$wave and the $2^{++}$wave are the same (and thus the same as in the E852 analysis).
While not shown here, the same is also true for the $1^{-+}$ and $2^{-+}$waves.

\begin{tabular}{|c|c|c|c|c|c|}
\hline$\pi_{2}(1670)$ & $\bar{M}$ & $=0^{+}$ & $\bar{M}$ & $=1$ & $M^{\epsilon}=1$ \\
\hline Decay & $\mathrm{L}$ & $\mathrm{H}$ & $\mathrm{L}$ & $\mathrm{H}$ & L $\quad \mathrm{H}$ \\
\hline$\left(f_{2} \pi\right)_{S}$ & $x$ & $x$ & $\times$ & $x$ & $x$ \\
\hline$\left(f_{2} \pi\right)_{D}$ & $x$ & $x$ & $x$ & $x$ & \\
\hline$\left[(\pi \pi)_{S}\right]_{D}$ & $\times$ & $x$ & & $x$ & \\
\hline$(\rho \pi)_{P}$ & $x$ & $x$ & & $x$ & \\
\hline$(\rho \pi)_{F}$ & & $x$ & & $x$ & \\
\hline$\left(f_{0} \pi\right)_{D}$ & & $x$ & & $x$ & \\
\hline
\end{tabular}

TABLE XII. The included decays of the $\pi_{2}(1670)$ in two analyses of the $3 \pi$ final state. "L" is the wave set used in the E852 analysis [76, 77. " $\mathrm{H}$ " is the wave set used in the higher statistics analysis [85].

E852-IU carried out a study to determine which of the additional waves in their "high wave" set were absorbing the intensity of the $\pi_{1}(1600)$. They found that the majority of this was due to the inclusion of the $\rho \pi$ decay of the $\pi_{2}(1670)$. The partial waves associated with the $\pi_{2}(1670)$ in both analyses are listed in Table XII. While the production from $M^{\epsilon}=0^{+}$is similar for both analyses, the E852 analysis only included the $\pi_{2}(1670)$ decaying to $f_{2} \pi$ in the $M^{\epsilon}=1^{+}$production. The highstatistics analysis included both $f_{2} \pi$ and $\rho \pi$ in both production mechanisms. The PDG [1] lists the two main decays of the $\pi_{2}(1670)$ as $f_{2} \pi(56 \%)$ and $\rho \pi(31 \%)$, so it seems odd to not include this latter decay in an analysis including the $\pi_{2}(1670)$. Figure 15 shows the results of removing the $\rho \pi$ decay from the "high wave" set for the $\pi^{+} \pi^{-} \pi^{-}$final state. This decay absorbs a significant portion of the $\pi_{1}(1600)$ partial wave.

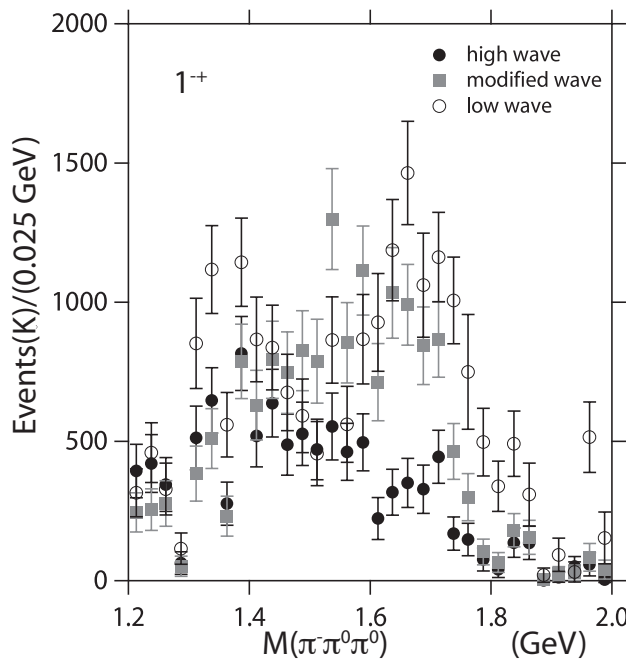

FIG. 15. The $1^{-+}$intensity for the charged mode for the high-wave set (filled circles), the modified high-wave set (filled squares), and the low-wave set (open circles). In the modified high-wave set the two $\rho \pi$ decays of the $\pi_{2}(1670)$ were removed from the fit. (Figure reproduced from reference [85.) 
In the E852-IU analyses, the fact that the phase motion of the $1^{-+}$exotic wave relative to other partial waves agrees with with those differences as measured by E852, and are the same in both the high-wave and low-wave analyses is intriguing. This could be interpreted as a $\pi_{1}(1600)$ state which is simply absorbed by the stronger $\pi_{2}(1670)$ as more partial waves are added. However, given the small actual phase difference between the $1^{-+}$ and $2^{-+}$partial waves (see Figure 11), the opposite conclusion is also possible, particularly if some small nonzero background phase were present. Here, the $1^{-+}$signal is due to leakage from the stronger $\pi_{2}$ and no $\pi_{1}(1600)$ is needed in the $\rho \pi$ final state.

The VES results have been summarized in a review of all their work on hybrid mesons 64. This included an updated summary of the $\pi_{1}(1600)$ in all four final states, $\eta^{\prime} \pi \rho \pi, b_{1} \pi$ and $f_{1} \pi$. In the $\eta^{\prime} \pi$ final state (Figure 16), they note that the $1^{-+}$wave is dominant. While they were concerned about the nature of the higher-mass part of the $2^{++}$spectrum $\left(a_{2}(1700)\right.$ or background) they find that a resonant description of $\pi_{1}(1600)$ is possible in both cases. For the case of the $b_{1} \pi$ final state (Figure 17), they find that the contribution of a $\pi_{1}(1600)$ resonance is required. In a combined fit to both the $\eta^{\prime} \pi$ and $b_{1} \pi$ data, they find a mass of $1.56 \pm 0.06 \mathrm{GeV}$ and a width of $0.34 \pm 0.06 \mathrm{GeV}$ for the $\pi_{1}(1600)$. In the $f_{1} \pi$ final state (Figure 18), they find a resonant description of the the $\pi_{1}(1600)$ with a mass of $1.64 \pm 0.03 \mathrm{GeV}$ and a width of $0.24 \pm 0.06 \mathrm{GeV}$ which they note is compatible with their measurement in the previous two final states. They also note, that in contradiction with E852 82, they find no significant $1^{-+}$intensity above a mass of $1.9 \mathrm{GeV}$ (see Section IIID. For the $\rho \pi$ final state, they are unable to conclude that the $\pi_{1}(1600)$ is present.

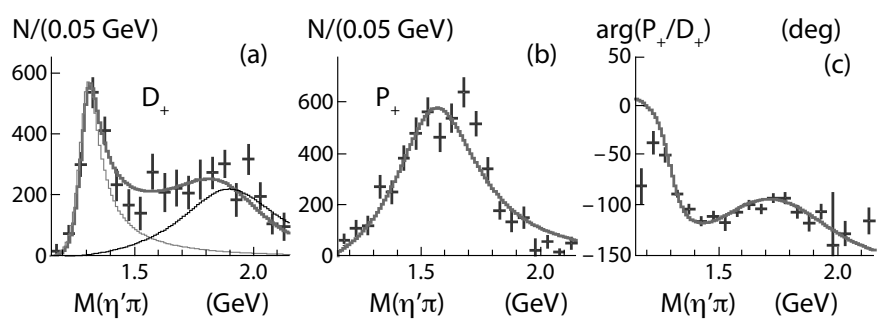

FIG. 16. The results of a partial wave analysis on the $\eta^{\prime} \pi^{-}$ final state from VES. (a) shows he $2^{++}$partial wave in $\omega \rho$, (b) shows the $1^{-+}$partial wave in $b_{1} \pi$ and (c) shows the interference between them. (Figure reproduced from reference 64.)

They note that the partial-wave analysis of the $\pi^{+} \pi^{-} \pi^{-}$system finds a significant contribution from the $J^{P C}=1^{+}$wave in the $\rho \pi$ channel (2 to $3 \%$ of the total intensity). Some of the models in the partial-wave analysis of the exotic wave lead to the appearance of a peak near a mass of $1.6 \mathrm{GeV}$ which resembles the $\pi_{1}(1600)$. However, the dependence of the size of this peak on the model used is significant 79 . They note that because the significance of the wave depends very strongly on the assumptions of coherence used in the analysis, the results

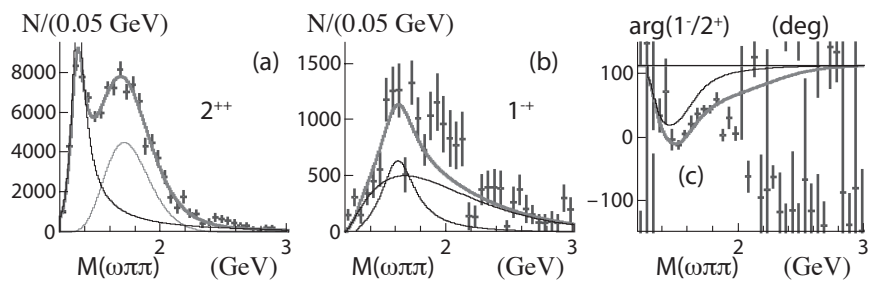

FIG. 17. The results of a partial wave analysis on the $b_{1} \pi$ final state from VES. (a) shows he $2^{++}$partial wave, (b) shows the $1^{-+}$partial wave and (c) shows the interference between them. (Figure reproduced from reference [64.)

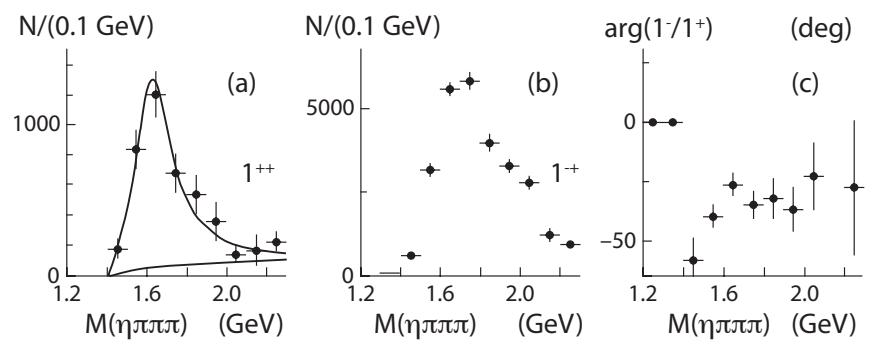

FIG. 18. The results of a partial wave analysis on the $f_{1} \pi$ final state from VES. (a) shows he $1^{++}$partial wave, (b) shows the $1^{-+}$partial wave and (c) shows the interference between them. (Figure reproduced from reference [64].)

for $3 \pi$ final states on the nature of the $\pi_{1}(1600)$ are not reliable.

To obtain a limit on the branching fraction of $\pi_{1}(1600)$ decay to $\rho \pi$, they looked at their results of the production of the $\pi_{1}(1600)$ in the charge-exchange reaction to $\eta^{\prime} \pi^{0}$ versus that of the $\eta^{\prime} \pi^{-}$final state. They conclude that the presence of the $\pi_{1}(1600)$ in $\eta^{\prime} \pi^{-}$and its absence in $\eta^{\prime} \pi^{0}$ preclude the formation of the $\pi_{1}(1600)$ by $\rho$ exchange. From this, they obtain the relative branching ratios for the $\pi_{1}(1600)$ as given in equation 12 .

$$
b_{1} \pi: f_{1} \pi: \rho \pi: \eta^{\prime} \pi=1.0 \pm .3: 1.1 \pm .3:<.3: 1
$$

While the results on $\rho \pi$ between E852 and VES seem at odds, we believe that these discrepancies are the result of the assumptions made in the analyses. These assumptions then manifest themselves in the interpretation of the results. The VES analyses fit both the real and imaginary parts of their amplitudes independently. However, for analytic functions, the two parts are not independent. Not using these constraints can lead to results that may be unphysical, and at a minimum, are discarding important constraints on the amplitudes. All of which can lead to difficulties in interpreting the results. In E852, many of their results rely on the assumption of a flat background phase, but there are many examples where this is not true. Thus, their results are biased towards a purely resonant description of the data, rather than a combination of resonant and non-resonant parts. It is also somewhat disappointing that E852 is unable to make statements about relative decay rates, or carry out 
a coupled channel analysis of their many data sets. Our understanding is that this is due to issues in modelling the rather tight trigger used in collecting their data.

The CLAS experiment at Jefferson Lab studied the reaction $\gamma p \rightarrow \pi^{+} \pi^{+} \pi^{-}(n)_{\text {miss }}$ to look for the production of the $\pi_{1}(1600)$ [86. The photons were produced by bremsstrahlung from a $5.7 \mathrm{GeV}$ electron beam. While there was significant contributions from Baryon resonances in their data, they attempted to remove this by selective cuts on various kinematic regions. The results of their partial-wave analysis show clear signals for the $a_{1}(1270)$, the $a_{2}(1320)$ and the $\pi_{2}(1670)$, but show no evidence for the $\pi_{1}(1600)$ decaying into three pions. They place and upper limit of the production and subsequent decay of the $\pi_{1}(1600)$ to be less than $2 \%$ of the $a_{2}(1320)$. There results imply that the $\pi_{1}(1600)$ is not strongly produced in photoproduction, the $\pi_{1}(1600)$ does not decay to $3 \pi$, or both.

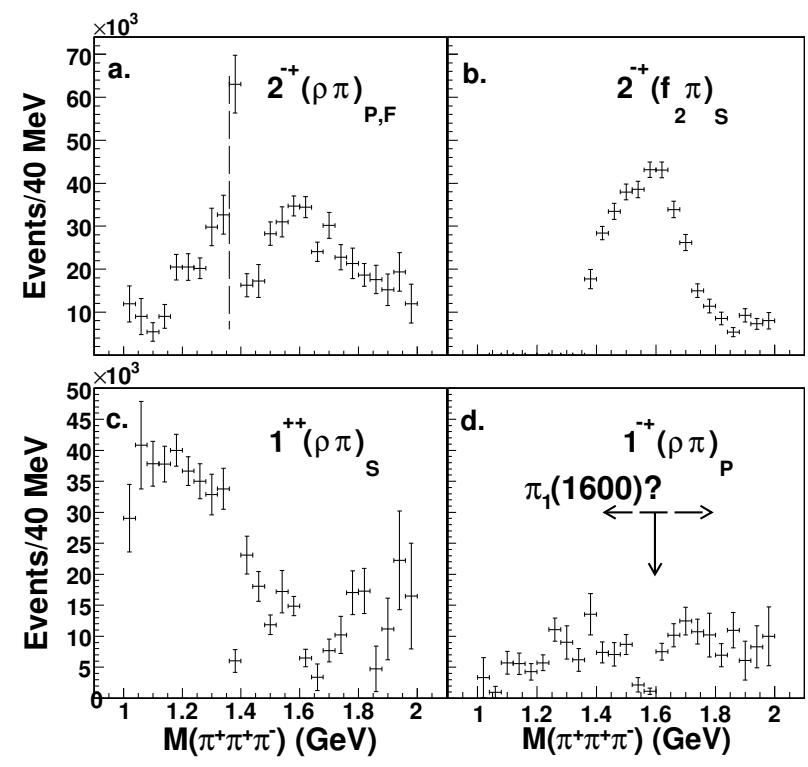

FIG. 19. The results from CLAS of a partial-wave analysis photoproduction data of the $3 \pi$ final state. Intensity is seen in the $2^{-+}$partial wave, (a) and (b), as well as the $1^{++}$partial wave (c). In the $1^{-+}$exotic wave, (d), no intensity is observed. (Figure reproduced from reference [86].)

The COMPASS experiment has reported their first study of the diffractively produced $3 \pi$ final state $[87,88$. They used a $190 \mathrm{GeV} / \mathrm{c}$ beam of pions to study the reaction $\pi^{-} P b \rightarrow \pi^{-} \pi^{-} \pi^{+} X$. In their partial-wave analysis of the $3 \pi$ final state, they observed the $\pi_{1}(1600)$ with a mass of $1.660 \pm 0.010_{-0.064}^{+0} \mathrm{GeV}$ and a width of $0.269 \pm 0.021_{-0.064}^{+0.042} \mathrm{GeV}$. The $\pi_{1}(1600)$ was produced dominantly in natural parity exchange $\left(M^{\epsilon}=1^{+}\right)$although unnatural parity exchange also seemed to be required. However, the level was not reported. The wave set (in reference 88]) used appears to be somewhat larger than that used in the high-statistics study of E852-IU [85. Thus, in the COMPASS analysis, the $\rho \pi$ decay of the $\pi_{2}(1670)$ does not appear to absorb the exotic intensity in their analysis. They also report on varying the rank of the fit with the $\pi_{1}(1600)$ and the results being robust against these changes. One point of small concern is that the mass and width that they extract for the $\pi_{1}(1600)$ are essentially identical to those for the $\pi_{2}(1670)$. For the latter, they observed a mass of $1.658 \pm 0.002_{-0.008}^{+0.024} \mathrm{GeV}$ and a width of $0.271 \pm 0.009_{-0.024}^{+0.022} \mathrm{GeV}$. However, the strength of the exotic wave appears to be about $20 \%$ of the $\pi_{2}$, thus feed through seems unlikely. Results from their partial-wave analysis are shown in Figures 20 and 21. These show the $1^{-+}$partial wave and the phase difference between the $1^{-+}$and $2^{-+}$waves. The solid curves are the results of mass-dependent fits to the $\pi_{1}(1600)$ and $\pi_{2}(1670)$.

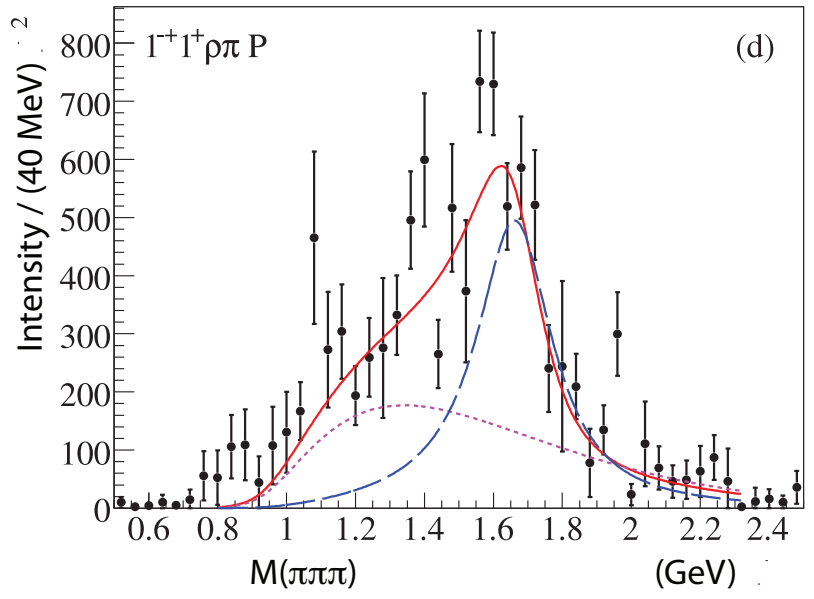

FIG. 20. (Color on line.) COMPASS results showing the intensity of the exotic $1^{-+}$wave. The solid (red) curve shows a fit to the corresponding resonances. The dashed (blue) curve is the $\pi_{1}(1600)$ while the dotted (magenta) curve is background. (Figure reproduced from reference [87].)

Table XIII summarizes the masses and widths found for the $\pi_{1}(1600)$ in the four decay modes and from the experiments which have seen a positive result. While the $\eta^{\prime} \pi, f_{1} \pi$ and $b_{1} \pi$ decay modes appear to be robust in the observation of a resonant $\pi_{1}(1600)$, there are concerns about the $3 \pi$ final states. While we report these in the table, the results should be taken with some caution.

Models for hybrid decays predict rates for the decay of the $\pi_{1}$. Equation 9 gives the predictions from reference [33. A second model from reference 34] predicted the following rates for a $\pi_{1}(1600)$.

$$
\begin{array}{ccccccc} 
& \pi b_{1} & \rho \pi & \pi f_{1} & \eta(1295) \pi & K^{*} K \\
\text { PSS } & 24 & 9 & 5 & 2 & 0.8 \\
\text { IKP } & 59 & 8 & 14 & 1 & 0.4
\end{array}
$$

These can be compared to the results from VES in equation 12, which are in moderate agreement. The real identification of the $\pi_{1}(1600)$ as a hybrid will almost certainly involve the identification of other members of the nonet: the $\eta_{1}$ and/or the $\eta_{1}^{\prime}$, both of which are expected to have 


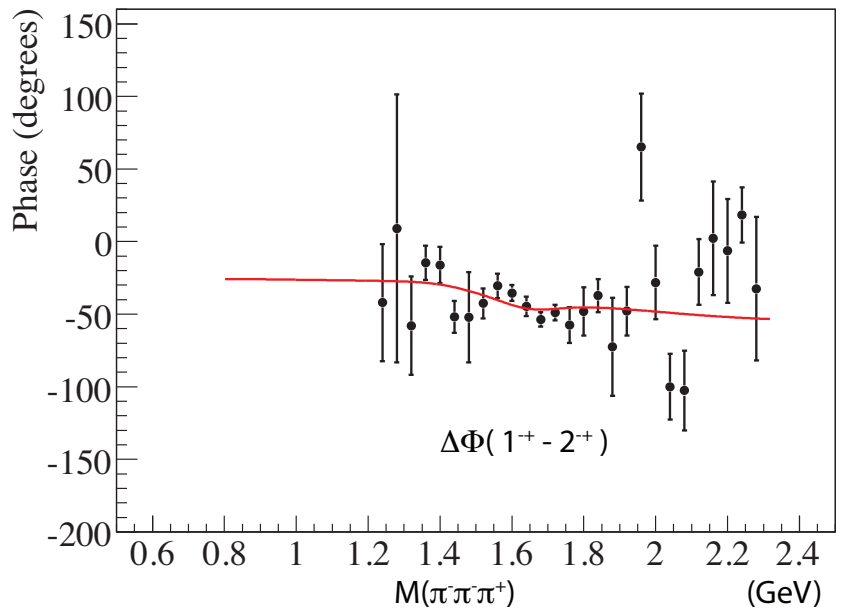

FIG. 21. (Color on line.) COMPASS results showing the phase difference between the exotic $1^{-+}$wave and the $2^{-+}$ wave. The solid (red) curve shows a fit to the corresponding resonances. (Figure reproduced from reference [87.)

\begin{tabular}{cllcr}
\hline \hline Mode & Mass $(\mathrm{GeV})$ & Width $(\mathrm{GeV})$ & Experiment & Reference \\
\hline$\rho \pi$ & $1.593 \pm 0.08$ & $0.168 \pm 0.020$ & E852 & {$[6]$} \\
$\eta^{\prime} \pi$ & $1.597 \pm 0.010$ & $0.340 \pm 0.040$ & E852 & {$[80$} \\
$f_{1} \pi$ & $1.709 \pm 0.024$ & $0.403 \pm 0.080$ & E852 & {$[82$} \\
$b_{1} \pi$ & $1.664 \pm 0.008$ & $0.185 \pm 0.025$ & E852 & {$[83$} \\
$b_{1} \pi$ & $1.58 \pm 0.03$ & $0.30 \pm 0.03$ & VES & {$[84$} \\
$b_{1} \pi$ & $1.61 \pm 0.02$ & $0.290 \pm 0.03$ & VES & {$[78$} \\
$b_{1} \pi$ & $\sim 1.6$ & $\sim 0.33$ & VES & 63 \\
$b_{1} \pi$ & $1.56 \pm 0.06$ & $0.34 \pm 0.06$ & VES & 64 \\
$f_{1} \pi$ & $1.64 \pm 0.03$ & $0.24 \pm 0.06$ & VES & 64 \\
$\eta^{\prime} \pi$ & $1.58 \pm 0.03$ & $0.30 \pm 0.03$ & VES & {$[84$} \\
$\eta^{\prime} \pi$ & $1.61 \pm 0.02$ & $0.290 \pm 0.03$ & VES & 78 \\
$\eta^{\prime} \pi$ & $1.56 \pm 0.06$ & $0.34 \pm 0.06$ & VES & 64 \\
$b_{1} \pi$ & $\sim 1.6$ & $\sim 0.23$ & CBAR & {$[81$} \\
$\rho \pi$ & $1.660 \pm 0.010$ & $0.269 \pm 0.021$ & COMPASS & {$[87$} \\
all & $1.662_{-0.011}^{+0.015}$ & $0.234 \pm 0.050$ & PDG & {$[1]$} \\
\hline \hline
\end{tabular}

TABLE XIII. Reported masses and widths of the $\pi_{1}(1600)$ from the E852 experiment, the VES experiment and the COMPASS experiment. The PDG average from 2008 is also reported.

widths that are similar to the $\pi_{1}$. For the case of the $\eta_{1}$, the most promising decay mode may be the $f_{1} \eta$ as it involves reasonably narrow daughters.

We believe that the current data support the existence of a resonant $\pi_{1}(1600)$ which decays into $b_{1} \pi, f_{1} \pi$ and $\eta^{\prime} \pi$, however, near-term confirmation of these results by COMPASS would be useful. For the $\rho \pi$ decay, we are uncertain. As noted earlier, the phase motion results observed by both E852 and E852-IU are can be interpreted as either the $\pi_{2}(1670)$ absorbing the $\pi_{1}(1600)$, or leakage from the $\pi_{2}(1670)$ generating a spurious signal in the $1^{-+}$channel. While the new COMPASS result are indeed interesting, we are concerned about their findings of exactly the same mass and width for the $\pi_{2}(1670)$ and the $\pi_{1}(1600)$. We are also concerned that their initial analyses may be over simplified, particularly in their bias towards an all-resonant description of their data. We hope that follow-on results from COMPASS will more broadly explore the model space imposed by their analyses. We would also like to see results on other final states coupled to those on three pions.

\section{The $\pi_{1}(2015)$}

The E852 experiment has also reported a third $\pi_{1}$ state seen decaying to both $f_{1} \pi$ [82] and to $b_{1} \pi$ [83. In the $f_{1} \pi$ final state, the $\pi_{1}(2015)$ is produced with $M^{\epsilon}=1^{+}$ in conjunction with the $\pi_{1}(1600)$. The description of the $1^{-+}$partial wave requires two poles. They report a mass of $2.001 \pm 0.030 \pm 0.092 \mathrm{GeV}$ and a width of $0.333 \pm$ $0.052 \pm 0.049 \mathrm{GeV}$. Figure 22 shows the E852 data from this final state. Parts $e$ and $f$ of this show the need for the two-pole solution. VES also examined the $f_{1} \pi$ final state, and their intensity of the $1^{-+}$partial wave above $1.9 \mathrm{GeV}$ (see Figure 18) is not inconsistent with that of E852 64. However, VES made no comment on this, nor have they claimed the existence of the $\pi_{1}(2015)$.

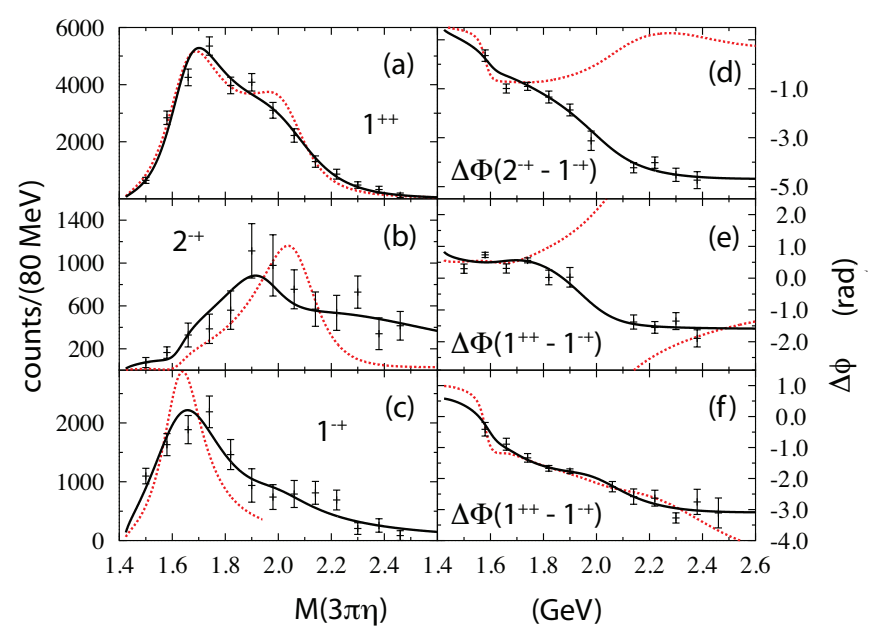

FIG. 22. (Color on line.) The $f_{1} \pi$ invariant mass from E852 82. (a) The $1^{++}$partial wave $\left(a_{1}(1270)\right)$, (b) the $2^{-+}$ partial wave $\left(\pi_{2}(1670)\right)$ and $(c)$ the exotic $1^{-+}$partial wave. The dotted (red) curves show the fits of Breit-Wigner distributions to the partial waves. (d) shows the phase difference between the $2^{-+}$and $1^{-+}$partial waves, while (e) shows the difference between the $1^{++}$and $1^{-+}$partial waves. The dotted (red) curves show the results for a single $\pi_{1}$ state, the $\pi_{1}(1600)$. (f) shows the same phase difference as in (d), but the dotted (red) curve shows a fit with two poles in the $1^{-+}$ partial wave, the $\pi_{1}(1600)$ and the $\pi_{1}(2015)$. (Figure reproduced from reference 82 .)

In the $b_{1} \pi$ final state, the $\pi_{1}(2015)$ is produced dominantly through natural parity exchange $\left(M^{\epsilon}=1^{+}\right)$while the $\pi_{1}(1600)$ was reported in both natural and unnatural parity exchange, where the unnatural exchange dominated. They observe a mass of $2.014 \pm 0.020 \pm 0.016 \mathrm{GeV}$ 


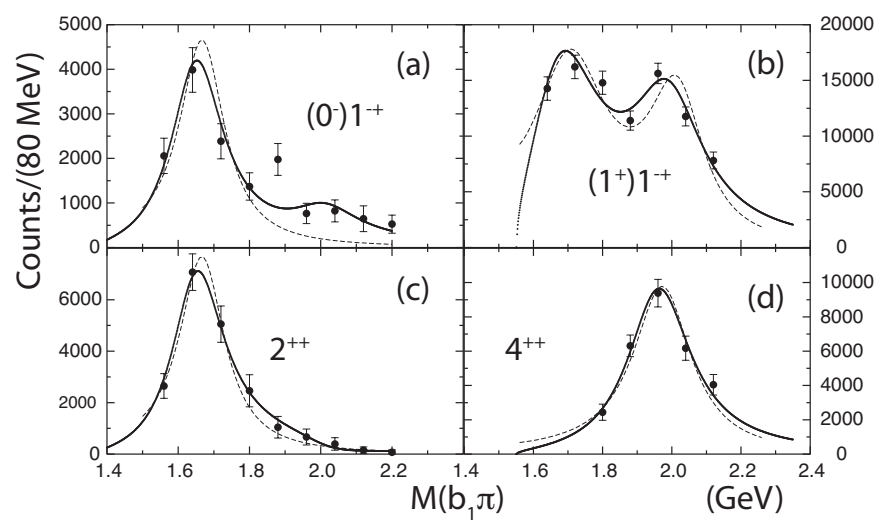

FIG. 23. The $b_{1} \pi$ invariant mass from the E852 experiment. (a) shows the $1^{-+} b_{1} \pi$ partial wave produced in natural parity exchange $\left(M^{\epsilon}=1^{+}\right)$while (b) shows the $1^{-+} b_{1} \pi$ partial wave produced in unnatural parity exchange $\left(M^{\epsilon}=0^{-}\right)$. In (c) is shown the $2^{++} \omega \rho$ partial wave, while (d) shows the $4^{++} \omega \rho$ partial wave. The curves are fits to the $\pi_{1}(1600)$ and $\pi_{1}(2015)$ (a and b), the $a_{2}(1700)$ in (c) and the $a_{4}(2040)$ in (d). (Figure reproduced from reference 83.)

and a width of $0.230 \pm 0.032 \pm 0.073 \mathrm{GeV}$ which are consistent with that observed in the $f_{1} \pi$ final state. Figure 23 shows the intensity distributions for several partial waves in this final states. The need for two states is most clearly seen in $b$. VES also looked at the $b_{1} \pi$ final state, but did not observe $1^{-+}$intensity above $1.9 \mathrm{GeV}$ 64]. However, the intensity shown in Figure 17 may be consistent with that observed by E852. The reported masses and widths are summarized in Table XIV] We note that this state does not appear in the summary tables of the PDG [1].

\begin{tabular}{crccc}
\hline \hline Mode & Mass $(\mathrm{GeV})$ & Width $(\mathrm{GeV})$ & Experiment & Reference \\
\hline$f_{1} \pi$ & $2.001 \pm 0.030$ & $0.333 \pm 0.052$ & $\mathrm{E} 852$ & 82 \\
$b_{1} \pi$ & $2.014 \pm 0.020$ & $0.230 \pm 0.032$ & $\mathrm{E} 852$ & 83 \\
\hline \hline
\end{tabular}

TABLE XIV. Reported masses and widths of the $\pi_{1}(2015)$ as observed in the E852 experiment. The PDG does not report an average for this state.

With so little experimental evidence for this high-mass state, it is difficult to say much. We note that the observed decays, $f_{1} \pi$ and $b_{1} \pi$ are those expected for a hybrid meson. We also note that the production of this state is consistent (natural parity exchange) for both of the observed final states. In the case that the $\pi_{1}(1600)$ is associated with the lowest-mass hybrid state, one possible interpretation of the $\pi_{1}(2015)$ would be a excited state (as suggested by recent LQCD calculations [29]). The mass splitting is typical of radial excitations observed in the normal mesons. In the case of the $\pi_{1}(1600)$ identified as something else, the $\pi_{1}(2015)$ would be a prime candidate for the lightest mass hybrid.

\section{E. Other Exotic-quantum Number States}

While no result has been published, the E852 collaboration has presented evidence at conferences for an isoscalar $2^{+-}$state 89 . The signal is observed with a mass near $1.9 \mathrm{GeV}$ in the $\omega \pi^{-} \pi^{+}$final state. It decays through $b_{1} \pi$ and is produced in both natural and unnatural parity exchange. This conference report was not followed up by a publication, so the signal should be viewed with caution. However, if confirmed, this state roughly lines up in mass with the $\pi_{1}(2015)$ and would be consistent with the lattice picture in which the $\pi_{1}(1600)$ is the lowest-mass hybrid and the $\pi_{1}(2015)$ is the first excitation [29].

\section{THE FUTURE}

The COMPASS experiment has recently started looking at pion peripheral production similar to work carried out by both VES and E852. Two new facilities are also expected in the not-too-distant future, PANDA at GSI and GlueX at Jefferson Lab. The former will study $\bar{p} p$ annihilation in the charmonium region, but it will also be possible to search for production of light-quark hybrids. GlueX will use a $9 \mathrm{GeV}$ beam of linearly polarized photons to produce hybrids.

Photoproduction of hybrids is interesting for several reasons. Simple arguments based on vector meson dominance suggest that the photon may behave like an $S=1$ $\bar{q} q$ system. In several models, such a system is more likely to couple to exotic quantum-number hybrids. Early calculations of hybrids used the apparent large $\rho \pi$ coupling of the $\pi_{1}(1600)$ to suggest that this state should be produced at least as strongly as normal mesons in photoproduction $90-92$. Unfortunately, the current controversy on the $\rho \pi$ decay of the $\pi_{1}(1600)$ makes the underlying assumption questionable, which may be confirmed by the non observation of the $\pi_{1}(1600)$ by CLAS [86].

Recently, lattice calculations have been performed to compute the radiative decay of charmonium $c \bar{c}$ and hybrid states 93 . In the charmonium system, they find that there is a large radiative decay for an exotic quantum number hybrid. These studies are currently being extended to the light-quark hybrids with the goal of providing estimates of the photoproduction cross sections of these states. However, based on the results in the charmonium sector, photoproduction appears to be a good place to look for hybrid mesons.

\section{CONCLUSIONS}

Over the last two decades, substantial data has been collected looking for exotic-quantum-number mesons. In particular, searches have focused on hybrid mesons, which arise due to excitations of the gluonic fields which 
confine quarks inside mesons. Models and LQCD predictions suggest that three nonets of exotic-quantumnumber states should exist, with $J^{P C}=0^{+-}, 1^{-+}$and $2^{+-}$, where the $1^{-+}$is expected to be the lightest. The most recent dynamical calculations of the isovector sector suggest a pair of $1^{-+}$states, with the $0^{+-}$and $2^{+-}$ states similar in mass to the heavier spin-one state. Calculations for the isoscalar states are currently underway, and preliminary results tend to agree with the isovector spectrum. Work is also underway to use lighter quark masses. These masses are measured by quoting the pion mass. Current work has pushed this to $390 \mathrm{MeV}$, and $260 \mathrm{MeV}$ is in progress. Calculations at the physical pion mass may be within reach.

While not supported by LQCD calculations, other models suggest that exotic-quantum-number multiquark states could exist as members of an $18 \oplus \overline{18}$ of SU(3). Expected $J^{P C}$ are $1^{-+}$and $0^{--}$, where the spin-one states are expected to be the lightest. The spin-zero states may be similar in mass. However, in order for these multiquark states to have finite widths, some additional binding mechanism needs to be present to prevent them from simply falling apart into pairs of mesons.

Measurements of the $J^{P C} \mathrm{~s}$, multiplet structure, and decays can be used to distinguish between these hybrid and multiquark states. However, to do this requires the observation of multiple members of a given multiplet as well as observation of states of different $J^{P C}$. Experimental results have provided evidence for three $J^{P C}=1^{-+}$isoscalar states, the $\pi_{1}(1400)$, the $\pi_{1}(1600)$ and the $\pi_{1}(2015)$.

The $\pi_{1}(1400)$ has been observed in both peripheral pion production and $\bar{p} n$ annihilation at rest. It has been seen decaying into $\eta \pi$ (in a p-wave), and even though other decay modes have been looked for (such has $\eta^{\prime} \pi$ and $\rho \pi$ ), no conclusive evidence for these has been found. While all experiments that have looked at the $\eta \pi$ final state agree that there is signal strength in the $1^{-+}$exotic wave, the interpretation of this signal is controversial. Explanations exist for the pion production data that describe the exotic wave as a non-resonant background phase, or produced by interference with non-resonant processes. Unfortunately, these explanations have not been tested against the $\bar{p} n$ data.

If the $\pi_{1}(1400)$ is resonant, it is difficult to explain it as a hybrid meson. It mass is too low, and its single decay appears inconsistent with state being part of an $\mathrm{SU}(3)$ nonet. Describing the $\pi_{1}(1400)$ as a multiquark state is a more natural explanation. However, in reviewing all the experimental evidence, as well as the non-resonant descriptions of the $1^{-+}$signal, we feel that the $\pi_{1}(1400)$ is not resonant.

The most extensive experimental evidence is for the $\pi_{1}(1600)$. It has been observed in four different decay modes, $\eta^{\prime} \pi, b_{1} \pi, f_{1} \pi$ and $\rho \pi$, by several experiments. Consistent results between E852 and VES are found for the first three decay modes, and from $\bar{p} p$ annihilation in flight for the $b_{1} \pi$ mode. However, the $\rho \pi$ decay is contro- versial. This mode has been observed by two groups, but not by two others. In one (VES), the strength is reported in the exotic wave, but they are unable to confirm that it is resonant. However, because not all physical constraints were used, their conclusions may be weaker than their data would suggest. A second group, E852-IU, explains the $\pi_{1}(1600)$ as feed through from the stronger $\pi_{2}(1670)$ state. However, while the intensity of the $\pi_{1}(1600)$ does depend on the decays of the $\pi_{2}(1670)$, the phase difference between the two states does not. This can be interpreted as either feed through from the $\pi_{2}(1670)$, or a resonant $\pi_{1}(1600)$ being absorbed by the $\pi_{2}(1670)$. To resolve this controversy will likely require a multi-channel analysis in which physics beyond a simple isobar picture is included. Even with this controversy about the $\rho \pi$ decay mode, we feel that the experimental evidence does support a resonant $\pi_{1}(1600)$. However, confirmation with higher statistics would be helpful.

Identification of the $\pi_{1}(1600)$ as the lightest hybrid is not inconsistent with both model predictions and LQCD calculations, although some might argue that its mass is somewhat low. The current observations and measurements are also consistent with a multiquark interpretation, although our feeling is that this is less likely. Observation of the isoscalar partners of this state would help to confirm its hybrid nature. Unfortunately, models predict their decays into channels that are experimentally difficult to analyze.

The evidence for the $\pi_{1}(2015)$ is much more limited. It has been seen by one experiment in two decay modes with very limited statistics while a second experiment (VES) does not see evidence for this state. What little is known about this state makes it a good candidate for a hybrid meson, but confirmation is clearly needed. If both the $\pi_{1}(1600)$ and the $\pi_{1}(2015)$ do exist, then the $\pi_{1}(2015)$ may be a radial excitation of the $\pi_{1}(1600)$. A result which is consistent with the most recent lattice calculations. As with the $\pi_{1}(1600)$, observation of the isoscalar partners to this state are important.

Beyond the $\eta_{1}$ and $\eta_{1}^{\prime}$ partners of the $\pi_{1}$ states, the crucial missing pieces of the hybrid puzzle are the other $J^{P C}$-exotic nonets, $0^{+-}$and $2^{+-}$. Here, there is a single hint of an $h_{2}$ state near $1.9 \mathrm{GeV}$, but no published results to this effect. As with the $\eta_{1}$ decays, those of these other nonets are also challenging, and to date, all the data that could be used in these searches has come from pion peripheral production. Definitive observation of these other nonets would provide the missing information to confirm the gluonic excitations of QCD. Fortunately, there will soon be four experimental programs running (COMPASS at CERN, BES III in Beijing, PANDA at GSI and GlueX at Jefferson Lab) that can provide new information on these issues. 


\section{ACKNOWLEDGMENTS}

The authors would like to thank Jozef Dudek for useful discussions and comments. This work was supported in part by the U.S. Department of Energy under grant No. DE-FG02-87ER40315.
[1] C. Amsler, et al. [The Particle Data Group], Phys. Lett. B 667, 1, (2008).

[2] T. Barnes and F. E. Close, Phys. Lett. B 116, 365 (1982).

[3] Eberhard Klempt and Alexander Zaitsev, Phys. Rep. 454, 1, (2007).

[4] V. Crede and C. A. Meyer, Prog. Part. Nucl. Phys. 63, $74,(2009)$.

[5] R. L. Jaffe and K. Johnson, Phys. Lett. B 60, 201, (1976).

[6] A. I. Vainshtein, M. B. Voloshin, V. I. Zakharov, V. A. Novikov, L. B. Okun and M. A. Shifman, Sov. J. Nucl. Phys. 27, 274 (1978) [Yad. Fiz. 27, 514 (1978)].

[7] T. Barnes, F. E. Close, F. de Viron and J. Weyers, Nucl. Phys. B 224, 241 (1983).

[8] M. S. Chanowitz and S. R. Sharpe, Nucl. Phys. B 222, 211 (1983) [Erratum-ibid. B 228, 588 (1983)].

[9] I. I. Balitsky, D. Diakonov and A. V. Yung, Phys. Lett. B 112, 71 (1982).

[10] J. I. Latorre, P. Pascual and S. Narison, Z. Phys. C 34, 347, (1987).

[11] S. Narison, Nucl. Phys. A 675, 54c, (2000).

[12] S. Narison, Phys. Lett. B 675, 319, (2009).

[13] Y. Nambu, Univ. of Chicago report No. 70-07, (1970).

[14] T. Nambu, Sci. Am. 235, No.5, 48, (1976).

[15] G. Bali et al. [SESAM Collaboration], Nucl. Phys. Proc. Suppl. 63, 209, (1998).

[16] N. Isgur and J. Paton, Phys. Rev. D 31, 2910, (1985).

[17] N. Isgur, R. Kokoski and J. Paton, Phys Rev. Lett. 54, 869, (1985).

[18] S. R. Cotanch and F. J. Llanes-Estrada, Nucl. Phys. A 689, (2001).

[19] S. R. Cotanch, I. J. General and P. Wang, Eur. Phys. J. A 31, 656 (2007).

[20] P. Lacock et al., Phys. Lett. B 401, 309, (1997).

[21] C. Bernard, J. E. Hetrick, T. A. DeGrand, M. Wingate, C. DeTar, C. McNeile, S. Gottlieb, U. M. Heller, K. Rummukainen, B. Sugar and D. Toussaint, Phys. Rev. D 56, 7039, (1997).

[22] P. Lacock, K. Schilling [SESAM Collaboration], Nucl. Phys. Proc. Suppl. 73, 261, (1999).

[23] C. Bernard et al., Nucl. Phys. B(Proc. Suppl.) 73, 264, (1999).

[24] Z. H. Mei and X. Q. Luo, Int. J. Mod. Phys. A 18, 5713 (2003).

[25] J. N. Hedditch et al., Phys. Rev. D 72, 114507, (2005).

[26] C. Bernard, T. Burch, E. B. Gregory, D. Toussaint, C. DeTar, J. Osborn, Steven Gottlieb, U. M. Heller and R. Sugar, Phys. Rev. D 68, 074505, (2003).

[27] C. McNeile and C. Michael, [UKQCD Collaboration], Phys. Rev. D 73, 074506 (2006).

[28] J. J. Dudek, R. G. Edwards, M. J. Peardon, D. G. Richards and C. E. Thomas, [Hadron Spectrum Collaboration], Phys. Rev. Lett. 103, 262001, (2009).

[29] J. J. Dudek, R. G. Edwards, M. J. Peardon, D. G. Richards, C. E. Thomas, accepted for publication in Phys. Rev. D (2010), arXiv:1004.4930 [hep-ph].
[30] Jozef Dudek, private communication.

[31] E. .S. Ackleh, T. Barnes and E. S. Swanson, Phys. Rev. D 54, 6811, (1996).

[32] T. Barnes, F. E. Close, P. R. Page and E. S. Swanson, Phys. Rev. D 55, 4157, (1997).

[33] F. E. Close and P. R. Page, Nucl. Phys. B 443, 23, (1995).

[34] P. R. Page, E. S. Swanson and A. P. Szczepaniak, Phys. Rev. D 59, 034016 (1999).

[35] F. E. Close and C. E. Thomas, Phys. Rev. C 79, 045201 (2009).

[36] T. Barnes and E. S. Swanson, Phys. Rev. C 77, 055206 (2008).

[37] P. R. Page, Phys. Lett. B 402, 183 (1997).

[38] F. E. Close and J. J. Dudek, Phys. Rev. D 70, 094015 (2004).

[39] C. McNeile, C. Michael and P. Pennanen, Phys. Rev. D 65, 094505, (2002).

[40] T. J. Burns and F. E. Close, "Hybrid-meson properties in lattice QCD and flux-tube models," Phys. Rev. D 74, 034003, (2006).

[41] F. E. Close and H. J. Lipkin, Phys. Lett. B 196, 245 (1987).

[42] S. U. Chung, E. Klempt and J. G. Korner, Eur. Phys. J. A 15, 539 (2002).

[43] C. J. Morningstar and M. J. Peardon, Phys. Rev. D 60, 034509 (1999).

[44] D. P. Roy, J. Phys. G 30, R113 (2004).

[45] F. E. Close, "An Introduction to Quarks and Partons", Academic Press (1979).

[46] R. L. Jaffe, Phys. Rev. D 17, 1444 (1978).

[47] R. L. Jaffe, Phys. Rev. D 15, 281 (1977).

[48] R. L. Jaffe and F. E. Low, Phys. Rev. D 19, 2105, (1979).

[49] A. T. Aerts, P. J. Mulders and J. J. de Swart, Phys. Rev. D 21, 1370 (1980).

[50] I. J. General, P. Wang, S. R. Cotanch and F. J. LlanesEstrada, Phys. Lett. B 653, 216 (2007).

[51] H. X. Chen, A. Hosaka and S. L. Zhu, Phys. Rev. D 78, 054017 (2008).

[52] H. X. Chen, A. Hosaka and S. L. Zhu, Phys. Rev. D 78, 117502 (2008).

[53] N. Mathur et al., Phys. Rev. D 76, 114505 (2007).

[54] S. Prelovsek and D. Mohler, Phys. Rev. D 79, 014503 (2009).

[55] S. Prelovsek, T. Draper, C. B. Lang, M. Limmer, K. F. Liu, N. Mathur and D. Mohler, (2010), arXiv:1005.0948 [hep-lat].

[56] S. U. Chung and T. L. Trueman, Phys. Rev. D 11, 633 (1975).

[57] S. U. Chung et al., Phys. Rev. D 60, 092001, (1999).

[58] A. Binosi and L. Theussl, Comp. Phys. Comm. 161, 76 (2004).

[59] D. Alde et al., Phys. Lett. B 205, 397, (1988).

[60] W. D. Apel et al., Nucl. Phys. B 193, 269, (1981).

[61] H. Aoyagi et al, Phys. Lett. B 314, 246, (1993).

[62] G. M. Beladidze et al. [VES Collaboration], Phys. Lett. 
B 313, 276, (1993).

[63] V. Dorofeev, et al. [VES Collaboration], AIP Conf. Proc. 619, 143, (2002).

[64] D. V. Amelin et al., Phys. Atom. Nucl. 68, 359 (2005) [Yad. Fiz. 68, 388 (2005)].

[65] D. R. Thompson et al. [E852 Collaboration], Phys. Rev. Lett. 79, 1630 (1997).

[66] A. Abele et al. [The Crystal Barrel Collaboration], Phys. Lett. B 423, 175 (1998).

[67] A. Abele et al. [The Crystal Barrel Collaboration], Phys. Lett. B 446, 349 (1999).

[68] A. R. Dzierba et al., Phys. Rev. D 67, 094015, (2003).

[69] G. S. Adams et al. [E852 Collaboration], Phys. Lett. B 657, 27 (2007).

[70] P. Salvini et al. [Obelix Collaboration], Eur. Phys. J. C 35, 21, (2004).

[71] W. Duenweber and F. Meyer-Wildhagen, AIP Conf. Proc. 717, 388, (2004).

[72] A. Donnachie and P. R. Page, Phys. Rev. D 58, 114012 (1998).

[73] R. Zhang, Y. B. Ding, X. Q. Li and P. R. Page, Phys. Rev. D 65, 096005 (2002).

[74] A. P. Szczepaniak, M. Swat, A. R. Dzierba and S. Teige, Phys. Rev. Lett. 91, 092002 (2003).

[75] Yu. P. Gouz et al. [VES Collaboration.], AIP Conf. Proc. 272, 572 (1993).

[76] G. S. Adams et al. [E852 Collaboration], Phys. Rev. Lett. 81, 5760 (1998).

[77] S. U. Chung et al. [E852 Collaboration], Phys. Rev. D 65, 072001, (2002).

[78] Yu A. Khokholov, et al, [VES Collaboration], Nucl. Phys.
A 663, 596, (2000).

[79] A. Zaitsev, et al. [VES Collaboration], Nucl. Phys. A 675, 155c, (2000).

[80] E. I. Ivanov et al. [E852 Collaboration], Phys. Rev. Lett. 86, 3977 (2001).

[81] C. A. Baker et al., Phys. Lett. B 563, 140 (2003).

[82] J. Kuhn et al. [E852 Collaboration], Phys. Lett. B 595, 109 (2004).

[83] M. Lu et al. [E852 Collaboration], Phys. Rev. Lett. 94, 032002 (2005).

[84] V. Dorofeev [VES Collaboration], arXiv:hep-ex/9905002 (1999).

[85] A. R. Dzierba et al., Phys. Rev. D 73, 072001 (2006).

[86] M.Nozar et al. [CLAS Collaboration], Phys. Rev. Lett. 102, 102002, (2009).

[87] A. Alekseev et al. [The COMPASS Collaboration], Phys. Rev. Lett. 104, 241803, (2010).

[88] B. Grube et al. [The COMPASS Collaboration], (2010), arXiv:1002.1272 [hep-ex].

[89] G. S. Adams et al., [E852 Collaboration], J. Phys. Conf. Ser. 9, 136 (2005).

[90] A. V. Afanasev and A. P. Szczepaniak, Phys. Rev. D 61, 114008, (2000).

[91] A. P. Szczepaniak and M. Swat, Phys. Lett. B 516, 72 (2001).

[92] F. E. Close and J. J. Dudek, Phys. Rev. D 70, 094015, (2004).

[93] J. J. Dudek, R. G. Edwards and C. E. Thomas, Phys. Rev. D 79, 094504 (2009). 